\title{
Curved thin domains and parabolic equations
}

\author{
by \\ M. PRizzi (Trieste), M. Rinaldi (Novara) \\ and K. P. RYBAKOWski (Rostock)
}

Abstract. Consider the family

$$
\begin{array}{ll}
u_{t}=\Delta u+G(u), & t>0, x \in \Omega_{\varepsilon}, \\
\partial_{\nu_{\varepsilon}} u=0, & t>0, x \in \partial \Omega_{\varepsilon},
\end{array}
$$

of semilinear Neumann boundary value problems, where, for $\varepsilon>0$ small, the set $\Omega_{\varepsilon}$ is a thin domain in $\mathbb{R}^{l}$, possibly with holes, which collapses, as $\varepsilon \rightarrow 0^{+}$, onto a (curved) $k$-dimensional submanifold of $\mathbb{R}^{l}$. If $G$ is dissipative, then equation $\left(E_{\varepsilon}\right)$ has a global attractor $\mathcal{A}_{\varepsilon}$.

We identify a "limit" equation for the family $\left(E_{\varepsilon}\right)$, prove convergence of trajectories and establish an upper semicontinuity result for the family $\mathcal{A}_{\varepsilon}$ as $\varepsilon \rightarrow 0^{+}$.

1. Introduction. Let $\omega$ be a bounded domain in $\mathbb{R}^{l}$ with Lipschitz boundary. Consider the Neumann boundary value problem

$$
\begin{array}{ll}
u_{t}=\Delta u+G(u), & t>0, x \in \omega, \\
\partial_{\nu} u=0, & t>0, x \in \partial \omega,
\end{array}
$$

on $\omega$. Here, $\nu$ is the exterior normal vector field on $\partial \omega$ and $G: \mathbb{R} \rightarrow \mathbb{R}$ is a given nonlinearity satisfying appropriate growth and regularity conditions.

Now suppose that $\omega:=\Omega_{\varepsilon}$ where $\Omega_{\varepsilon}$ is a domain which depends on a small parameter $\varepsilon>0$. Intuitively, the set $\Omega_{\varepsilon}$ (or a part of it) is "thin" of order $\varepsilon$ and, as $\varepsilon \rightarrow 0^{+}$, the domain $\Omega_{\varepsilon}$ "degenerates" to some limit set, which may no longer be a domain in $\mathbb{R}^{l}$.

One may now naturally ask what happens to solutions of equation (1) on $\omega:=\Omega_{\varepsilon}$ as $\varepsilon \rightarrow 0^{+}$. Is there a limit equation and, if so, do some solutions of the limit equation persist for small $\varepsilon>0$ ?

One of the earliest papers devoted to such questions is the work [16] by Hale and Vegas. In that paper a bifurcation mechanism is described

2000 Mathematics Subject Classification: Primary 35K57, 35B25, 35B41; Secondary 35P15, 53B21.

Key words and phrases: curved squeezed domains, attractors, reaction-diffusion equations. 
which produces stable spatially nonhomogeneous stationary solutions of equation (1). The basic idea is simple: start with a (disconnected) set $\Omega_{0} \subset \mathbb{R}^{2}$ consisting of two disjoint disk-like open sets $B_{1}$ and $B_{2}$ and connect $B_{1}$ with $B_{2}$ by a small channel of thickness $\varepsilon>0$ to produce the two-dimensional dumbbell domain $\Omega_{\varepsilon}$. Suppose $a, b \in \mathbb{R}$ are two different zeros of the function $G$. Then the function $u: \Omega \rightarrow \mathbb{R}$ defined by $u(x) \equiv a$ on $B_{1}$ and $u(x) \equiv b$ on $B_{2}$ is an equilibrium of equation (1) on $\omega:=\Omega_{0}$. Under appropriate hypotheses on $G$ and the thin channel in $\Omega_{\varepsilon}$ it is shown in [16] that the equilibrium $u$ can be continued to a family of stable equilibria of equation (1) on $\omega:=\Omega_{\varepsilon}$, for $\varepsilon>0$ small.

The paper [16] gave rise to a number of other important articles on dumbbell type domains; see [2], [3], [18], [20] and the reference section in the survey [25] by Raugel.

Now suppose that $\omega=\Omega_{\varepsilon}$ is everywhere "thin" of order $\varepsilon>0$. Then the domain $\Omega_{\varepsilon}$ collapses, as $\varepsilon \rightarrow 0^{+}$, to a lower-dimensional set. Suppose also that the nonlinearity $G$ is dissipative so equation (1) has a global attractor $\mathcal{A}_{\varepsilon}$ on an appropriate phase space. In addition to the questions mentioned above one may now ask what happens to the family of attractors $\mathcal{A}_{\varepsilon}$ as $\varepsilon \rightarrow 0^{+}$.

This problem was first considered in [13] by Hale and Raugel for domains $\Omega_{\varepsilon}$ having the special form

$$
\Omega_{\varepsilon}=\left\{\left(x_{1}, x_{2}\right) \in \mathbb{R}^{l-1} \times \mathbb{R}=\mathbb{R}^{l} \mid x_{1} \in \widetilde{\omega} \text { and } 0<x_{2}<\varepsilon g\left(x_{1}\right)\right\},
$$

where $g$ is a smooth positive function defined on an $(l-1)$-dimensional domain $\widetilde{\omega}$. For dissipative nonlinearities they prove that the limit equation is the $(l-1)$-dimensional boundary value problem

$$
\begin{array}{ll}
u_{t}=(1 / g) \operatorname{div}(g \nabla u)+G(u), & t>0, x \in \widetilde{\omega}, \\
\partial_{\nu} u=0, & t>0, x \in \partial \widetilde{\omega} .
\end{array}
$$

They compare the semiflows generated by these equations and establish an important upper semicontinuity result for the corresponding family of attractors. For $l=2$, Hale and Raugel also prove existence of inertial manifolds containing these attractors. In a subsequent paper [14] the same authors establish an upper semicontinuity result for damped wave equations. See also [4], [11] and [25] for some other upper semicontinuity results for problems on thin domains.

In [21] (cf. also [22]), the first and the third authors of the present paper considered equation (1) on a class of thin domains which are much more general than those considered in [13], including, e.g., domains with holes. In this context they developed an abstract framework for the analysis of the questions mentioned above, based on a property of strong spectral convergence satisfied by the linear part of the equation. 
Let us describe in some detail the results of [21]. Let $k \in \mathbb{N}$ with $k<l$ and $\Omega$ be a nonempty bounded domain in $\mathbb{R}^{l}=\mathbb{R}^{k} \times \mathbb{R}^{l-k}$ with Lipschitz boundary. Write $x=\left(x_{1}, x_{2}\right)$ for a generic point of $\mathbb{R}^{k} \times \mathbb{R}^{l-k}$. Given $\varepsilon>0$ squeeze $\Omega$ by the factor $\varepsilon$ in the $y$-direction to obtain the flatly squeezed domain $\Omega_{\varepsilon}$. More precisely, let $T_{\varepsilon}: \mathbb{R}^{k} \times \mathbb{R}^{l-k} \rightarrow \mathbb{R}^{k} \times \mathbb{R}^{l-k}$ be the flat squeezing transformation $\left(x_{1}, x_{2}\right) \mapsto\left(x_{1}, \varepsilon x_{2}\right)$ and set $\Omega_{\varepsilon}:=T_{\varepsilon}(\Omega)$. Note that the domains considered by Hale and Raugel arise from the flat squeezing of the domain

$$
\Omega:=\left\{\left(x_{1}, x_{2}\right) \mid x_{1} \in \widetilde{\omega} \text { and } 0<x_{2}<g\left(x_{1}\right)\right\} .
$$

Under appropriate conditions on $G$, equation (1) on the varying domain $\omega:=\Omega_{\varepsilon}$ can then equivalently be described, in abstract terms, by the equation

$$
\dot{u}+\widetilde{A}_{\varepsilon} u=\widehat{G}(u)
$$

on $H^{1}\left(\Omega_{\varepsilon}\right)$. Here $\widetilde{A}_{\varepsilon}$ is the selfadjoint operator defined by the bilinear form

$$
\widetilde{a}_{\varepsilon}(u, v)=\int_{\Omega_{\varepsilon}} \nabla u \cdot \nabla v d x
$$

on $H^{1}\left(\Omega_{\varepsilon}\right)$ and $\widehat{G}$ is the Nemytskiu operator defined by the function $G$. Via the change of variables $u(x) \mapsto u(\widetilde{x})$, where $\widetilde{x}=T_{\varepsilon}(x)$, we can transform (3) to the equivalent equation

$$
\dot{u}+A_{\varepsilon} u=\widehat{G}(u)
$$

on the fixed space $H^{1}(\Omega)$. Here, $A_{\varepsilon}$ is the operator defined by the formula

$$
A_{\varepsilon}\left(u \circ T_{\varepsilon}\right)=\widetilde{A}_{\varepsilon}(u) \circ T_{\varepsilon} .
$$

Equation (4) defines a (local) semiflow $\pi_{\varepsilon}$ on $H^{1}(\Omega)$ which, for dissipative nonlinearities $G$, has a global attractor $\mathcal{A}_{\varepsilon}$.

Note that $A_{\varepsilon}$ is the linear operator induced by the bilinear form

$$
a_{\varepsilon}(u, v):=\int_{\Omega}\left(\nabla_{x_{1}} u \cdot \nabla_{x_{1}} v+\frac{1}{\varepsilon^{2}} \nabla_{x_{2}} u \cdot \nabla_{x_{2}} v\right) d x
$$

on $H^{1}(\Omega)$. Observe that for $u \in H^{1}(\Omega)$,

$$
\lim _{\varepsilon \rightarrow 0^{+}} a_{\varepsilon}(u, u)= \begin{cases}\int_{\Omega}\left|\nabla_{x_{1}} u\right|^{2} d x & \text { if } \nabla_{x_{2}} u=0 \\ +\infty & \text { otherwise }\end{cases}
$$

Thus the family $a_{\varepsilon}(u, u), \varepsilon>0$, of real numbers has a finite limit (as $\varepsilon \rightarrow 0^{+}$) if and only if $u \in H_{\mathrm{S}}^{1}(\Omega)$, where we define

$$
H_{\mathrm{s}}^{1}(\Omega):=\left\{u \in H^{1}(\Omega) \mid \nabla_{x_{2}} u=0\right\} .
$$

This is a closed linear subspace of $H^{1}(\Omega)$. The corresponding limit bilinear form is given by the formula

$$
a_{0}(u, v):=\int_{\Omega} \nabla_{x_{1}} u \cdot \nabla_{x_{1}} v d x, \quad u, v \in H_{\mathrm{s}}^{1}(\Omega) .
$$


The form $a_{0}$ uniquely determines a densely defined selfadjoint linear operator

$$
A_{0}: D\left(A_{0}\right) \subset H_{\mathrm{s}}^{1}(\Omega) \rightarrow L_{\mathrm{s}}^{2}(\Omega)
$$

by the usual formula

$$
a_{0}(u, v)=\left\langle A_{0} u, v\right\rangle_{L^{2}(\Omega)} \quad \text { for } u \in D\left(A_{0}\right) \text { and } v \in H_{\mathrm{s}}^{1}(\Omega) .
$$

Here, $L_{\mathrm{s}}^{2}(\Omega)$ is the closure of $H_{\mathrm{s}}^{1}(\Omega)$ in the $L^{2}$-norm, so $L_{\mathrm{s}}^{2}(\Omega)$ is a closed linear subspace of $L^{2}(\Omega)$. It turns out that, as $\varepsilon \rightarrow 0^{+}$, the operators $A_{\varepsilon}$ converge to the operator $A_{0}$ in some spectral sense and the linear semigroups $e^{-t A_{\varepsilon}}$ "singularly" converge to the semigroup $e^{-t A_{0}}$ in some strong sense.

We can now consider the abstract parabolic equation

$$
\dot{u}=-A_{0} u+\widehat{G}(u)
$$

on the space $H_{\mathrm{s}}^{1}(\Omega)$. This equation defines a (local) semiflow $\pi_{0}$ on $H_{\mathrm{s}}^{1}(\Omega)$ which, for dissipative $G$, has a global attractor $\mathcal{A}_{0}$. It turns out that, as $\varepsilon \rightarrow$ $0^{+}$, the family $\pi_{\varepsilon}$ of semiflows converges, in some strong singular sense, to the semiflow $\pi_{0}$. As a consequence we obtain, for dissipative nonlinearities $G$, an upper semicontinuity result for the resulting family $\left(\mathcal{A}_{\varepsilon}\right)_{\varepsilon \geq 0}$ of attractors, which extends the corresponding result of [13] to the present case. As proved in [23], the inertial manifold result from [13] also holds in this more general situation.

The analogues of the results of [21] for damped wave equations are proved in the recent paper [7], which, in particular, contains an extension of the upper semicontinuity result from [14]. Finally, some applications of Conley index to reaction-diffusion equations and damped wave equations on squeezed domains appear in [6] and [8].

Note that the above papers all deal with a rather special flat squeezing of $\Omega$ onto a lower dimensional subspace of $\mathbb{R}^{l}$ (cf. Figure 1 ).

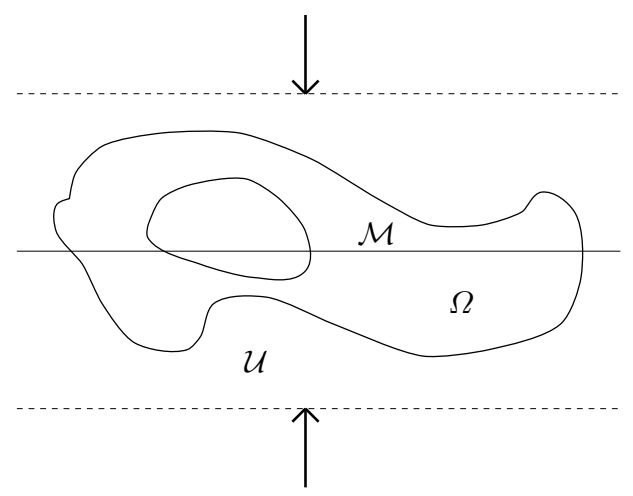

Fig. 1. Flat squeezing

It is geometrically much more appealing and also more realistic from the point of view of applications to consider general squeezing of the domain 
$\Omega$ onto a curved lower dimensional submanifold $\mathcal{M}$ of $\mathbb{R}^{l}$ and to study the effect of such squeezing upon the behavior of solutions of reaction-diffusion equations. This is the purpose of the present paper.

Let us briefly describe the geometry of the problem considered here. Let $l, k$ and $r$ be positive integers with $r \geq 2, l \geq 2$ and $k<l$. Let $\mathcal{M} \subset \mathbb{R}^{l}$ be an imbedded $k$-dimensional submanifold of $\mathbb{R}^{l}$ of class $C^{r}$. Note that, in the general case considered here, the manifold is global, i.e. $\mathcal{M}$ need not be included in a single coordinate chart. Let us also remark that we do not assume $\mathcal{M}$ to be orientable.

By the tubular neighborhood theorem there exists an open set $\mathcal{U}$ in $\mathbb{R}^{l}$ and a map $\phi: \mathcal{U} \rightarrow \mathcal{M}$ of class $C^{r-1}$ such that whenever $x \in \mathcal{U}$ and $p \in \mathcal{M}$ then $\phi(x)=p$ if and only if the vector $x-p$ is orthogonal to $T_{p} \mathcal{M}$; moreover, $\varepsilon x+(1-\varepsilon) \phi(x) \in \mathcal{U}$ for all $x \in \mathcal{U}$ and all $\varepsilon \in[0,1]$.

For $\varepsilon \in[0,1]$ let us define the curved squeezing transformation $\Phi_{\varepsilon}: \mathcal{U} \rightarrow \mathbb{R}^{l}$ by

$$
\Phi_{\varepsilon}(x):=\varepsilon x+(1-\varepsilon) \phi(x)=\phi(x)+\varepsilon(x-\phi(x)) .
$$

Now let $\Omega$ be an arbitrary nonempty bounded domain in $\mathbb{R}^{l}$ with Lipschitz boundary and such that $\mathrm{Cl} \Omega \subset \mathcal{U}$. For $\varepsilon \in] 0,1]$, define the curved squeezed domain $\Omega_{\varepsilon}:=\Phi_{\varepsilon}(\Omega)$. (Cf. Figure 2.)

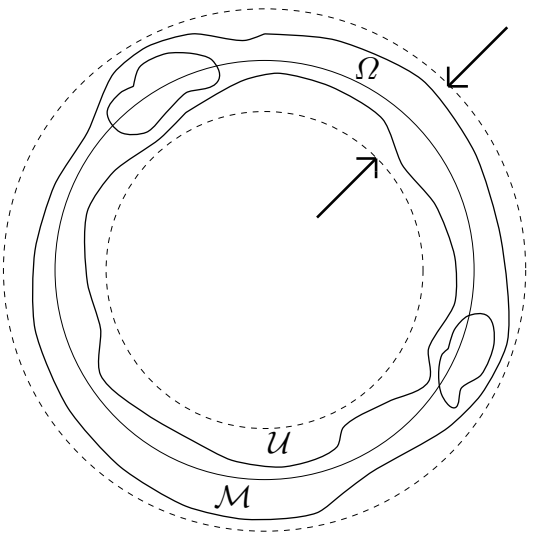

Fig. 2. Curvilinear squeezing

Note that setting, in particular, $\mathcal{M}:=\mathbb{R}^{k} \times\{0\}, \mathcal{U}:=\mathbb{R}^{k} \times \mathbb{R}^{l-k}$ and letting $\phi$ be the orthogonal projection $\left(x_{1}, x_{2}\right) \mapsto\left(x_{1}, 0\right)$ of $\mathbb{R}^{k} \times \mathbb{R}^{l-k}$ onto $\mathcal{M}$ we are reduced to the flat squeezing case described above.

Consider equation (1) on the curved squeezed domain $\omega:=\Omega_{\varepsilon}$. This equation can again be described in abstract terms as the equation

$$
\dot{u}+\widetilde{A}_{\varepsilon} u=\widehat{G}(u)
$$


on $H^{1}\left(\Omega_{\varepsilon}\right)$. Here, the operator $\widetilde{A}_{\varepsilon}$ is induced by the bilinear form

$$
\widetilde{a}_{\varepsilon}(u, v)=\int_{\Omega_{\varepsilon}} \nabla u \cdot \nabla v d x .
$$

We can now use the change of variables $u(x) \mapsto u(\widetilde{x})$, where $\widetilde{x}=\Phi_{\varepsilon}(x)$, to transform equation (7) to the equivalent problem

$$
\dot{u}+A_{\varepsilon} u=\widehat{G}(u)
$$

on $H^{1}(\Omega)$. Here, the operator $A_{\varepsilon}$ is defined by the formula

$$
A_{\varepsilon}\left(u \circ \Phi_{\varepsilon}\right)=\left(\widetilde{A}_{\varepsilon} u\right) \circ \Phi_{\varepsilon} .
$$

Equation (8) defines a (local) semiflow $\pi_{\varepsilon}$ on $H^{1}(\Omega)$, which, for dissipative $G$, has a global attractor $\mathcal{A}_{\varepsilon}$.

For $x \in \mathcal{U}$ denote by $Q(x): \mathbb{R}^{l} \rightarrow \mathbb{R}^{l}$ the orthogonal projection of $\mathbb{R}^{l} \cong T_{p} \mathbb{R}^{l}$ onto $T_{p} \mathcal{M}$, where $p:=\phi(x)$. Then $P(x):=I-Q(x)$ is the orthogonal projection onto the orthogonal complement of $T_{p} \mathcal{M}$.

Now define

$$
H_{\mathrm{s}}^{1}(\Omega):=\left\{u \in H^{1}(\Omega) \mid P(x) \nabla u(x)=0 \text { a.e. }\right\} .
$$

Note that $H_{\mathrm{s}}^{1}(\Omega)$ is a closed linear subspace of the Hilbert space $H^{1}(\Omega)$. Let $L_{\mathrm{s}}^{2}(\Omega)$ be the closure in $L^{2}(\Omega)$ of $H_{\mathrm{s}}^{1}(\Omega)$.

It is one of the main contributions of this paper to show that the family $\left(A_{\varepsilon}\right)_{\varepsilon \in] 0,1]}$ of operators converges in a strong spectral sense to a densely defined selfadjoint operator $A_{0}$ in $L_{\mathrm{s}}^{2}(\Omega)$ (cf. Sections 2 and 3 below).

Both the space $H_{\mathrm{s}}^{1}(\Omega)$ defined above and the limit operator $A_{0}$ defined in Section 3 below strongly depend on the geometry of $\mathcal{M}$ and especially on its curvature. The presence of curvature, reflected in the nonlinearity of the mapping $\Phi_{\varepsilon}$, renders the proof of the existence of $A_{0}$ much more involved than the corresponding proof in the flat squeezing case.

We can now consider the abstract parabolic equation

$$
\dot{u}+A_{0} u=\widehat{G}(u)
$$

on the space $H_{\mathrm{s}}^{1}(\Omega)$, where $H_{\mathrm{s}}^{1}(\Omega)$ is defined in (9). Equation (10) defines a (local) semiflow $\pi_{0}$ on $H_{\mathrm{s}}^{1}(\Omega)$, which, for dissipative $G$, has a global attractor $\mathcal{A}_{0}$.

We prove in Section 4 that, as $\varepsilon \rightarrow 0^{+}$, the linear semigroups $e^{-t A_{\varepsilon}}$ singularly converge to the semigroup $e^{-t A_{0}}$ and the semiflows $\pi_{\varepsilon}$ singularly converge to $\pi_{0}$. In the dissipative case, we also obtain an upper semicontinuity result for the family $\left(\mathcal{A}_{\varepsilon}\right)_{\varepsilon \in[0,1]}$ of attractors.

We thus obtain a far-reaching generalization of the results proved in [21] for the flat squeezing case. Further developments of this research will appear in [24], where some of the results given here are used to prove existence of inertial manifolds on some genuinely high dimensional thin domains. 
Lastly, it is worth mentioning that various thin domain problems have also been studied in the context of elasticity theory. This is a vast and fascinating subject for which we refer the reader to the monograph [10] by Ciarlet.

2. Properties of curved squeezing transformations. In this section we define curved squeezing transformations and discuss their fundamental properties. The results of this section are crucial for the rest of this paper.

We assume throughout that $l, k$ and $r$ are positive integers with $r \geq 2$, $l \geq 2$ and $k<l$. By $\langle\cdot, \cdot\rangle$ we denote the standard inner product in $\mathbb{R}^{l}$.

Let $\mathcal{M} \subset \mathbb{R}^{l}$ be an imbedded $k$-dimensional submanifold of $\mathbb{R}^{l}$ of class $C^{r}$. For $p \in \mathcal{M}$ we denote by $T_{p} \mathcal{M}$ the tangent space to $\mathcal{M}$ at the point $p$. We will identify $T_{p} \mathcal{M}$ with a subspace of $\mathbb{R}^{l}$.

Definition 2.1. An open set $\mathcal{U}$ in $\mathbb{R}^{l}$ is called a normal neighborhood of $\mathcal{M}$ if there is a map $\phi: \mathcal{U} \rightarrow \mathcal{M}$ of class $C^{r-1}$ such that:

(1) whenever $x \in \mathcal{U}$ and $p \in \mathcal{M}$ then $\phi(x)=p$ if and only if the vector $x-p$ is orthogonal to $T_{p} \mathcal{M}$;

(2) $\varepsilon x+(1-\varepsilon) \phi(x) \in \mathcal{U}$ for all $x \in \mathcal{U}$ and all $\varepsilon \in[0,1]$.

The following properties easily follow from the above definition:

Proposition 2.2. Let $\mathcal{U}$ be a normal neighborhood of $\mathcal{M}$. Then the map $\phi$ of Definition 2.1 is uniquely determined by item (1) of that definition. Moreover,

(1) $\phi(\mathcal{U})=\mathcal{M}$ and $\phi(x)=x$ if and only if $x \in \mathcal{M}$;

(2) $D \phi(x) \nu=0$ for all $x \in \mathcal{U}$ and all vectors $\nu$ orthogonal to $T_{p} \mathcal{M}$, where $p:=\phi(x)$.

Note that, by the tubular neighborhood theorem (see, e.g., [5]), a normal neighborhood of $\mathcal{M}$ always exists.

In what follows we consider a fixed normal neighborhood $\mathcal{U}$ of $\mathcal{M}$ and let the map $\phi$ be as in Definition 2.1. Recall that for $x \in \mathcal{U}$ we denote by $Q(x), P(x): \mathbb{R}^{l} \rightarrow \mathbb{R}^{l}$ the orthogonal projections onto $T_{p} \mathcal{M}$ and onto the orthogonal complement of $T_{p} \mathcal{M}$, where $p:=\phi(x)$. Here $P(x)=I-Q(x)$. by

For $\varepsilon \in[0,1]$ define the curved squeezing transformation $\Phi_{\varepsilon}: \mathcal{U} \rightarrow \mathbb{R}^{l}$

$$
\Phi_{\varepsilon}(x):=\varepsilon x+(1-\varepsilon) \phi(x)=\phi(x)+\varepsilon(x-\phi(x)) .
$$

The following properties are an immediate consequence of the definition:

Proposition 2.3. The map $[0,1] \times \mathcal{U} \rightarrow \mathbb{R}^{l},(\varepsilon, x) \mapsto \Phi_{\varepsilon}(x)$, is continuous. If $\varepsilon \in] 0,1]$, then: 
(1) $\Phi_{\varepsilon}(\mathcal{U})=\{y \in \mathcal{U} \mid \phi(y)+(1 / \varepsilon)(y-\phi(y)) \in \mathcal{U}\}, \Phi_{\varepsilon}(\mathcal{U})$ is open in $\mathbb{R}^{l}$ and $\Phi_{\varepsilon}: \mathcal{U} \rightarrow \Phi_{\varepsilon}(\mathcal{U})$ is a diffeomorphism of class $C^{r-1}$ with

$$
\Phi_{\varepsilon}^{-1}(y)=\phi(y)+(1 / \varepsilon)(y-\phi(y)), \quad y \in \Phi_{\varepsilon}(\mathcal{U}) ;
$$

(2) $\phi\left(\Phi_{\varepsilon}(x)\right)=\phi(x)$ for $x \in \mathcal{U}$.

The following result is of crucial importance for the whole paper:

Theorem 2.4. For $x \in \mathcal{U}$ and $\varepsilon \in[0,1]$ define

$$
J_{\varepsilon}(x):= \begin{cases}\varepsilon^{-(l-k) / 2}\left|\operatorname{det} D \Phi_{\varepsilon}(x)\right| & \text { if } \varepsilon>0, \\ \mid \operatorname{det}\left(\left.D \phi(x)\right|_{\left.T_{\phi(x)} \mathcal{M}\right)} \mid\right. & \text { otherwise. }\end{cases}
$$

Then

$$
J_{\varepsilon}(x)>0 \quad \text { for all } \varepsilon \in[0,1] \text { and } x \in \mathcal{U} .
$$

Moreover, the function $[0,1] \times \mathcal{U} \rightarrow \mathbb{R},(\varepsilon, x) \mapsto J_{\varepsilon}(x)$, is continuous.

For every $\varepsilon \in[0,1]$ and $x \in \mathcal{U}$ there exists a linear map $S_{\varepsilon}(x): \mathbb{R}^{l} \rightarrow \mathbb{R}^{l}$ such that, for $\varepsilon \in] 0,1]$,

$$
D \Phi_{\varepsilon}^{-1}\left(\Phi_{\varepsilon}(x)\right)=S_{\varepsilon}(x)+(1 / \varepsilon) P(x) \quad \text { for all } x \in \mathcal{U} .
$$

Accordingly,

$$
\left(D \Phi_{\varepsilon}^{-1}\left(\Phi_{\varepsilon}(x)\right)\right)^{T}=S_{\varepsilon}(x)^{T}+(1 / \varepsilon) P(x) \quad \text { for all } x \in \mathcal{U} .
$$

The following properties are satisfied:

(1) The maps $[0,1] \times \mathcal{U} \rightarrow \mathcal{L}\left(\mathbb{R}^{l}, \mathbb{R}^{l}\right)$,

$$
(\varepsilon, x) \mapsto S_{\varepsilon}(x) \quad \text { and } \quad(\varepsilon, x) \mapsto S_{\varepsilon}(x)^{T},
$$

are continuous;

(2) for every $\varepsilon \in[0,1], x \in \mathcal{U}$ and $\nu$ orthogonal to $T_{\phi(x)} \mathcal{M}$,

$$
S_{\varepsilon}(x) \nu=S_{\varepsilon}(x)^{T} \nu=0 ;
$$

(3) for every $\varepsilon \in[0,1]$ and $x \in \mathcal{U}$ the maps

$$
\left.S_{\varepsilon}(x)\right|_{T_{\phi(x)} \mathcal{M}}: T_{\phi(x)} \mathcal{M} \rightarrow T_{\phi(x)} \mathcal{M},\left.\quad S_{\varepsilon}(x)^{T}\right|_{T_{\phi(x)} \mathcal{M}}: T_{\phi(x)} \mathcal{M} \rightarrow T_{\phi(x)} \mathcal{M}
$$

are well defined and bijective. Furthermore,

$$
\left(\left.S_{0}(x)\right|_{T_{\phi(x)} \mathcal{M}}\right)^{-1}=\left.D \phi(x)\right|_{T_{\phi(x)} \mathcal{M}}, \quad\left(\left.S_{0}(x)^{T}\right|_{T_{\phi(x)} \mathcal{M}}\right)^{-1}=\left.D \phi(x)^{T}\right|_{T_{\phi(x)} \mathcal{M}}
$$

Finally, $\phi: \mathcal{U} \rightarrow \mathcal{M}$ is an open map.

The rest of this section is devoted to the proof of Theorem 2.4. We begin with an obvious local result:

Proposition 2.5. For every $p \in \mathcal{M}$ there is an open set $V_{p}$ in $\mathcal{M}$ and $C^{r-1}$-maps $h_{i}=h_{p, i}: V_{p} \rightarrow \mathbb{R}^{l}, i=1, \ldots, k, \nu_{j}=\nu_{p, j}: V_{p} \rightarrow \mathbb{R}^{l}$ and $\alpha_{j}=\alpha_{p, j}: \phi^{-1}\left(V_{p}\right) \rightarrow \mathbb{R}, j=1, \ldots, l-k$, such that for every $q \in V_{p}$ the vectors $h_{i}(q), i=1, \ldots, k$, form an orthonormal basis of $T_{q} \mathcal{M}$, and the vectors 
$\nu_{j}(q), j=1, \ldots, l-k$, form an orthonormal basis of the orthogonal complement of $T_{q} \mathcal{M}$ in $T_{q} \mathbb{R}^{l} \cong \mathbb{R}^{l}$. Moreover, for $x \in \phi^{-1}\left(V_{p}\right), y \in \Phi_{\varepsilon}\left(\phi^{-1}\left(V_{p}\right)\right)$ and $h \in \mathbb{R}^{l}$,

$$
\begin{aligned}
Q(x) h & =\sum_{i=1}^{k}\left\langle h, h_{i}(\phi(x))\right\rangle h_{i}(\phi(x)), \\
P(x) h & =\sum_{j=1}^{l-k}\left\langle h, \nu_{j}(\phi(x))\right\rangle \nu_{j}(\phi(x)), \\
x & =\phi(x)+\sum_{j=1}^{l-k} \alpha_{j}(x) \nu_{j}(\phi(x)) \\
\Phi_{\varepsilon}(x) & =\phi(x)+\varepsilon \sum_{j=1}^{l-k} \alpha_{j}(x) \nu_{j}(\phi(x)), \\
\Phi_{\varepsilon}^{-1}(y) & =\phi(y)+(1 / \varepsilon) \sum_{j=1}^{l-k} \alpha_{j}(y) \nu_{j}(\phi(y)) .
\end{aligned}
$$

Since $\phi: \mathcal{U} \rightarrow \mathcal{M}$, we have of course $D \phi(x) h \in T_{\phi(x)} \mathcal{M}$ for all $x \in \mathcal{U}$ and $h \in \mathbb{R}^{l}$. In particular,

$$
\left.D \phi(x)\right|_{T_{\phi(x)} \mathcal{M}}: T_{\phi(x)} \mathcal{M} \rightarrow T_{\phi(x)} \mathcal{M}
$$

Now let $p \in \mathcal{M}$ and let the set $V_{p}$ and the maps $h_{i}, \nu_{j}$ and $\alpha_{j}$ be as in Proposition 2.5. Since $\nu_{j}(q)$ has norm one for all $q \in V_{p}$ and $j=1, \ldots, l-k$, differentiating the equality

$$
\left\langle\nu_{j}(\phi(x)), \nu_{j}(\phi(x))\right\rangle=1, \quad x \in \phi^{-1}\left(V_{p}\right),
$$

we obtain

$$
\left\langle D \nu_{j}(\phi(x)) D \phi(x) h, \nu_{j}(\phi(x))\right\rangle=0
$$

for all $x \in \phi^{-1}\left(V_{p}\right), h \in \mathbb{R}^{l}$ and $j=1, \ldots, l-k$.

Differentiating the identity

$$
x=\phi(x)+\sum_{j=1}^{l-k} \alpha_{j}(x) \nu_{j}(\phi(x)), \quad x \in \phi^{-1}\left(V_{p}\right),
$$

we get

$$
h=D \phi(x) h+\sum_{j=1}^{l-k}\left(D \alpha_{j}(x) h\right) \nu_{j}(\phi(x))+\sum_{j=1}^{l-k} \alpha_{j}(x) D \nu_{j}(\phi(x)) D \phi(x) h
$$

for all $x \in \phi^{-1}\left(V_{p}\right)$ and $h \in \mathbb{R}^{l}$. 
For $x \in \phi^{-1}\left(V_{p}\right)$ and $h \in T_{\phi(x)} \mathcal{M}$ we thus obtain

$$
h=D \phi(x) h+\sum_{j=1}^{l-k} \alpha_{j}(x) Q(x) D \nu_{j}(\phi(x)) D \phi(x) h .
$$

For $x \in \phi^{-1}\left(V_{p}\right)$, set

$$
S_{0}(x) h:=Q(x) h+\sum_{j=1}^{l-k} \alpha_{j}(x) Q(x) D \nu_{j}(\phi(x)) Q(x) h, \quad h \in \mathbb{R}^{l} .
$$

Then $\left.S_{0}(x)\right|_{T_{\phi(x)} \mathcal{M}}: T_{\phi(x)} \mathcal{M} \rightarrow T_{\phi(x)} \mathcal{M}$ and using (18)-(20) we obtain

$$
\left(\left.S_{0}(x)\right|_{T_{\phi(x)} \mathcal{M}}\right) \circ\left(\left.D \phi(x)\right|_{T_{\phi(x)} \mathcal{M}}\right)=I_{T_{\phi(x)} \mathcal{M}}
$$

for all $x \in \phi^{-1}\left(V_{p}\right)$. Since $T_{\phi(x)} \mathcal{M}$ is finite-dimensional, this implies that $\left.D \phi(x)\right|_{T_{\phi(x)} \mathcal{M}}$ is bijective and

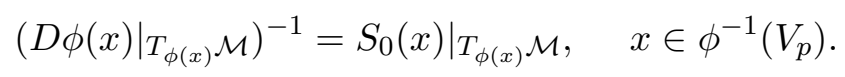

Since $p \in \mathcal{M}$ is arbitrary, we obtain

$$
J_{0}(x)>0, \quad x \in \mathcal{U}
$$

We also find that $D \phi(x): \mathbb{R}^{l} \rightarrow T_{\phi(x)}(\mathcal{M})$ is surjective for all $x \in \mathcal{U}$. By the surjective mapping theorem (cf. [1, Theorem 3.5.2]) we conclude that $\phi: \mathcal{U} \rightarrow \mathcal{M}$ is an open map.

Now let $\varepsilon \in] 0,1]$. Formula (11) and Proposition 2.3 imply, for all $x \in \mathcal{U}$, $y \in \Phi_{\varepsilon}(\mathcal{U})$ and every $h \in \mathbb{R}^{l}$,

$$
\begin{aligned}
D \Phi_{\varepsilon}(x) h & =D \phi(x) h+\varepsilon(h-D \phi(x) h), \\
D \Phi_{\varepsilon}^{-1}(y) h & =D \phi(y) h+(1 / \varepsilon)(h-D \phi(y) h) .
\end{aligned}
$$

This immediately implies that

$$
\begin{aligned}
\left.D \Phi_{\varepsilon}(x)\right|_{T_{\phi(x)} \mathcal{M}}: T_{\phi(x)} \mathcal{M} & \rightarrow T_{\phi(x)} \mathcal{M} \\
\left.D \Phi_{\varepsilon}^{-1}(y)\right|_{T_{\phi(y)} \mathcal{M}}: T_{\phi(y)} \mathcal{M} & \rightarrow T_{\phi(y)} \mathcal{M}
\end{aligned}
$$

Thus, using Proposition 2.5 we obtain, for $x \in \phi^{-1}\left(V_{p}\right)$ and $h \in T_{\phi(x)} \mathcal{M}$,

$$
D \Phi_{\varepsilon}(x) h=D \phi(x) h+\varepsilon \sum_{j=1}^{l-k} \alpha_{j}(x) Q(x) D \nu_{j}(\phi(x)) D \phi(x) h .
$$

For $y \in \Phi_{\varepsilon}\left(\phi^{-1}\left(V_{p}\right)\right)$ and $h \in T_{\phi(y)} \mathcal{M}$ we get

$$
D \Phi_{\varepsilon}^{-1}(y) h=D \phi(y) h+(1 / \varepsilon) \sum_{j=1}^{l-k} \alpha_{j}(y) Q(y) D \nu_{j}(\phi(y)) D \phi(y) h .
$$

Moreover, for $x \in \phi^{-1}\left(V_{p}\right)$ and $y \in \Phi_{\varepsilon}\left(\phi^{-1}\left(V_{p}\right)\right)$ we also obtain

$$
D \Phi_{\varepsilon}(x) \nu_{j}(\phi(x))=\varepsilon \nu_{j}(\phi(x))
$$




$$
D \Phi_{\varepsilon}^{-1}(y) \nu_{j}(\phi(y))=(1 / \varepsilon) \nu_{j}(\phi(y))
$$

for $j=1, \ldots, l-k$.

For $x \in \phi^{-1}\left(V_{p}\right)$, we also have

$$
\left|\operatorname{det} D \Phi_{\varepsilon}(x)\right|=\left(\operatorname{det} G_{\varepsilon}(x)\right)^{1 / 2}
$$

where $G_{\varepsilon}(x)$ is the symmetric $l \times l$ matrix whose entries are

$$
G_{\varepsilon}(x)_{i j}= \begin{cases}\left\langle D \Phi_{\varepsilon}(x) h_{i}(\phi(x)), D \Phi_{\varepsilon}(x) h_{j}(\phi(x))\right\rangle, & 1 \leq i, j \leq k, \\ \left\langle D \Phi_{\varepsilon}(x) \nu_{i-k}(\phi(x)), D \Phi_{\varepsilon}(x) \nu_{j-k}(\phi(x))\right\rangle, & k+1 \leq i, j \leq l, \\ \left\langle D \Phi_{\varepsilon}(x) \nu_{i-k}(\phi(x)), D \Phi_{\varepsilon}(x) h_{j}(\phi(x))\right\rangle, & k+1 \leq i \leq l, 1 \leq j \leq k .\end{cases}
$$

Hence, by (29) and (25), we get

$$
\left|\operatorname{det} D \Phi_{\varepsilon}(x)\right|=\varepsilon^{(l-k) / 2}\left(\operatorname{det} \widetilde{G}_{\varepsilon}(x)\right)^{1 / 2}>0,
$$

where $\widetilde{G}_{\varepsilon}(x)$ is the $k \times k$ matrix whose entries are

$$
\widetilde{G}_{\varepsilon}(x)_{i j}=\left\langle D \Phi_{\varepsilon}(x) h_{i}(\phi(x)), D \Phi_{\varepsilon}(x) h_{j}(\phi(x))\right\rangle .
$$

Thus, for every $x \in \phi^{-1}\left(V_{p}\right)$,

$$
J_{\varepsilon}(x)= \begin{cases}\left(\operatorname{det}\left(\left\langle D \Phi_{\varepsilon}(x) h_{i}(\phi(x)), D \Phi_{\varepsilon}(x) h_{j}(\phi(x))\right\rangle\right)_{i j}\right)^{1 / 2}>0, & \varepsilon \in] 0,1], \\ \left(\operatorname{det}\left(\left\langle D \phi(x) h_{i}(\phi(x)), D \phi(x) h_{j}(\phi(x))\right\rangle\right)_{i j}\right)^{1 / 2}>0, & \varepsilon=0 .\end{cases}
$$

Since $p \in \mathcal{M}$ is arbitrary we therefore obtain all the statements of Theorem 2.4 concerning $J_{\varepsilon}$.

Next we analyze the linear operator

$$
L_{\varepsilon}(x):=D \Phi_{\varepsilon}^{-1}\left(\Phi_{\varepsilon}(x)\right)
$$

for $\varepsilon \in] 0,1]$. Formula (30) implies

$$
L_{\varepsilon}(x) \nu_{j}(\phi(x))=(1 / \varepsilon) \nu_{j}(\phi(x)) \quad \text { for } x \in \phi^{-1}\left(V_{p}\right) \text { and } j=1, \ldots, l-k .
$$

Moreover, (28) and Proposition 2.5 imply, for $x \in \phi^{-1}\left(V_{p}\right)$ and $h \in T_{\phi(x)} \mathcal{M}$,

$$
\begin{aligned}
L_{\varepsilon}(x) h= & D \phi\left(\Phi_{\varepsilon}(x)\right) h \\
& +(1 / \varepsilon) \sum_{j=1}^{l-k} \alpha_{j}\left(\Phi_{\varepsilon}(x)\right) Q\left(\Phi_{\varepsilon}(x)\right) D \nu_{j}\left(\phi\left(\Phi_{\varepsilon}(x)\right)\right) D \phi\left(\Phi_{\varepsilon}(x)\right) h \\
= & D \phi\left(\Phi_{\varepsilon}(x)\right) h+\sum_{j=1}^{l-k} \alpha_{j}(x) Q(x) D \nu_{j}(\phi(x)) D \phi\left(\Phi_{\varepsilon}(x)\right) h .
\end{aligned}
$$

Set

$$
B_{\varepsilon}(x):=D \phi\left(\Phi_{\varepsilon}(x)\right), \quad N_{j}(x):=D \nu_{j}(\phi(x))
$$

Then

$$
L_{\varepsilon}(x) h=B_{\varepsilon}(x) h+\sum_{j=1}^{l-k} \alpha_{j}(x) Q(x) N_{j}(x) B_{\varepsilon}(x) h
$$


for $x \in \phi^{-1}\left(V_{p}\right)$ and $h \in T_{\phi(x)} \mathcal{M}$. Thus, for $x \in \phi^{-1}\left(V_{p}\right)$ and $h \in \mathbb{R}^{l}$, we get

$$
\begin{aligned}
L_{\varepsilon}(x) h= & Q(x) B_{\varepsilon}(x) Q(x) h \\
& +\sum_{j=1}^{l-k} \alpha_{j}(x) Q(x) N_{j}(x) B_{\varepsilon}(x) Q(x) h+(1 / \varepsilon) P(x) h .
\end{aligned}
$$

(Here we used the fact that $B_{\varepsilon}(x)=D \phi\left(\Phi_{\varepsilon}(x)\right)$ maps $\mathbb{R}^{l}$ onto $T_{\phi(x)} \mathcal{M}$, so $B_{\varepsilon}(x)=Q(x) B_{\varepsilon}(x)$ for $x \in \mathcal{U}$.)

Now observe that, since $\Phi_{\varepsilon}(x) \rightarrow \phi(x)$ as $\varepsilon \rightarrow 0$ in $\mathbb{R}^{l}$, uniformly on compact subsets of $\mathcal{U}$, we also have

$$
B_{\varepsilon}(x)=D \phi\left(\Phi_{\varepsilon}(x)\right) \rightarrow D \phi(\phi(x)) \quad \text { as } \varepsilon \rightarrow 0
$$

in $\mathcal{L}\left(\mathbb{R}^{l}, \mathbb{R}^{l}\right)$, uniformly on compact subsets of $\mathcal{U}$. Since $\phi(x) \equiv x$ on $\mathcal{M}$, we have $D \phi(\phi(x)) h=h$ for all $x \in \mathcal{U}$ and all $h \in T_{\phi(x)} \mathcal{M}$. Moreover, whenever $\nu$ is orthogonal to $T_{\phi(x)} \mathcal{M}$ then $D \phi(\phi(x)) \nu=0$. It follows that

$$
D \phi(\phi(x))=Q(x) \quad \text { for } x \in \mathcal{U},
$$

and hence

$$
B_{\varepsilon}(x) \rightarrow Q(x) \quad \text { as } \varepsilon \rightarrow 0
$$

in $\mathcal{L}\left(\mathbb{R}^{l}, \mathbb{R}^{l}\right)$, uniformly on compact subsets of $\mathcal{U}$. For $x \in \mathcal{U}$ set

$$
S_{\varepsilon}(x):=L_{\varepsilon}(x)-(1 / \varepsilon) P(x) .
$$

It follows that $\left.S_{\varepsilon}(x)\right|_{T_{\phi(x)} \mathcal{M}}: T_{\phi(x)} \mathcal{M} \rightarrow T_{\phi(x)} \mathcal{M}$ and $\left.S_{\varepsilon}(x)\right|_{T_{\phi(x)} \mathcal{M}}=$ $\left.L_{\varepsilon}(x)\right|_{T_{\phi(x)} \mathcal{M}}$. This implies that $\left.S_{\varepsilon}(x)\right|_{T_{\phi(x)} \mathcal{M}}: T_{\phi(x)} \mathcal{M} \rightarrow T_{\phi(x)} \mathcal{M}$ is bijective. Moreover, using (33), we see that, for $x \in \phi^{-1}\left(V_{p}\right)$,

$$
S_{\varepsilon}(x)=Q(x) B_{\varepsilon}(x) Q(x)+\sum_{j=1}^{l-k} \alpha_{j}(x) Q(x) N_{j}(x) B_{\varepsilon}(x) Q(x) .
$$

Therefore

$$
\left|S_{\varepsilon}(x)-S_{0}\left(x_{0}\right)\right|_{\mathcal{L}\left(\mathbb{R}^{l}, \mathbb{R}^{l}\right)} \rightarrow 0 \quad \text { as } \varepsilon \rightarrow 0 \text { and } x \rightarrow x_{0} \text { in } \phi^{-1}\left(V_{p}\right)
$$

where

$$
S_{0}(x)=Q(x)+\sum_{j=1}^{l-k} \alpha_{j}(x) Q(x) N_{j}(x) Q(x)
$$

is the linear operator defined in (20). We recall that, by (21), $\left.S_{0}(x)\right|_{T_{\phi(x)} \mathcal{M}}$ : $T_{\phi(x)} \mathcal{M} \rightarrow T_{\phi(x)} \mathcal{M}$ is bijective and $\left(\left.S_{0}(x)^{T}\right|_{T_{\phi(x)} \mathcal{M}}\right)^{-1}=\left.D \phi(x)^{T}\right|_{T_{\phi(x)} \mathcal{M}}$.

We now have to compute $L_{\varepsilon}(x)^{T}$. Since $P(x)$ and $Q(x)$ are orthogonal projections in $\mathbb{R}^{l}$, we have $P(x)^{T}=P(x)$ and $Q(x)^{T}=Q(x)$ for all $x \in \mathcal{U}$. 
For $x \in \phi^{-1}\left(V_{p}\right)$ we have

$$
\begin{aligned}
& S_{\varepsilon}(x)^{T}=Q(x) B_{\varepsilon}(x)^{T} Q(x)+\sum_{j=1}^{l-k} \alpha_{j}(x) Q(x) B_{\varepsilon}(x)^{T} N_{j}(x)^{T} Q(x), \\
& S_{0}(x)^{T}=Q(x)+\sum_{j=1}^{l-k} \alpha_{j}(x) Q(x) N_{j}(x)^{T} Q(x) .
\end{aligned}
$$

It follows that $S_{\varepsilon}(x)^{T} \nu_{j}(\phi(x))=0$ and $S_{0}(x)^{T} \nu_{j}(\phi(x))=0$ for $j=1, \ldots$, $l-k$. We also have

$$
\begin{aligned}
& \left\langle S_{\varepsilon}(x)^{T} h, \nu_{j}(\phi(x))\right\rangle=\left\langle h, S_{\varepsilon}(x) \nu_{j}(\phi(x))\right\rangle=0, \\
& \left\langle S_{0}(x)^{T} h, \nu_{j}(\phi(x))\right\rangle=\left\langle h, S_{0}(x) \nu_{j}(\phi(x))\right\rangle=0
\end{aligned}
$$

for all $x \in \phi^{-1}\left(V_{p}\right), h \in \mathbb{R}^{l}$ and $j=1, \ldots, l-k$. It follows that

$$
\left.S_{\varepsilon}(x)^{T}\right|_{T_{\phi(x)} \mathcal{M}}: T_{\phi(x)} \mathcal{M} \rightarrow T_{\phi(x)} \mathcal{M},\left.\quad S_{\varepsilon}(x)^{T}\right|_{T_{\phi(x)} \mathcal{M}}=\left.L_{\varepsilon}(x)^{T}\right|_{T_{\phi(x)} \mathcal{M}}
$$

This implies that $\left.S_{\varepsilon}(x)^{T}\right|_{T_{\phi(x)}} \mathcal{M}: T_{\phi(x)} \mathcal{M} \rightarrow T_{\phi(x)} \mathcal{M}$ is bijective. Moreover, $\left.S_{0}(x)^{T}\right|_{T_{\phi(x)} \mathcal{M}}: T_{\phi(x)} \mathcal{M} \rightarrow T_{\phi(x)} \mathcal{M}$ and

$$
\left|S_{\varepsilon}(x)^{T}-S_{0}\left(x_{0}\right)^{T}\right|_{\mathcal{L}\left(\mathbb{R}^{l}, \mathbb{R}^{l}\right)} \rightarrow 0 \quad \text { as } \varepsilon \rightarrow 0 \text { and } x \rightarrow x_{0} \text { in } \phi^{-1}\left(V_{p}\right) .
$$

Now given $x \in \mathcal{U}, h \in T_{\phi(x)} \mathcal{M}$ and $\nu$ orthogonal to $T_{\phi(x)} \mathcal{M}$ we have

$$
\left\langle D \phi(x)^{T} h, \nu\right\rangle=\langle h, D \phi(x) \nu\rangle=0,
$$

so $\left.D \phi(x)^{T}\right|_{T_{\phi(x)} \mathcal{M}}: T_{\phi(x)} \mathcal{M} \rightarrow T_{\phi(x)} \mathcal{M}$. Finally, observe that, by (21), for all $h, h^{\prime} \in T_{\phi(x)} \mathcal{M}$ we have

$$
\left\langle D \phi(x)^{T} S_{0}(x)^{T} h, h^{\prime}\right\rangle=\left\langle h, S_{0}(x) D \phi(x) h^{\prime}\right\rangle=\left\langle h, h^{\prime}\right\rangle .
$$

It follows that $\left(\left.D \phi(x)^{T}\right|_{\left.T_{\phi(x)} \mathcal{M}\right)}\right)\left(\left.S_{0}(x)^{T}\right|_{T_{\phi(x)} \mathcal{M}}\right)=\left.I\right|_{T_{\phi(x)} \mathcal{M}}$. This implies that $\left.S_{0}(x)^{T}\right|_{T_{\phi(x)} \mathcal{M}}$ is bijective and

$$
\left(\left.S_{0}(x)^{T}\right|_{T_{\phi(x)} \mathcal{M}}\right)^{-1}=\left.D \phi(x)^{T}\right|_{T_{\phi(x)} \mathcal{M}}
$$

The proof of Theorem 2.4 is complete.

3. Spectral convergence. For the rest of this paper let $\Omega$ be a nonempty open bounded set in $\mathbb{R}^{l}$ with Lipschitz boundary. Suppose that $\mathrm{Cl} \Omega \subset$ $\mathcal{U}$. For $\varepsilon \in] 0,1]$ let $\Omega_{\varepsilon}:=\Phi_{\varepsilon}(\Omega)$ be the squeezed domain. In this section we will study the bilinear forms on $H^{1}\left(\Omega_{\varepsilon}\right)$ stemming from the Laplacian on $\Omega_{\varepsilon}$ with Neumann boundary conditions. We will show that, as $\varepsilon \rightarrow 0^{+}$, these bilinear forms tend in a strong spectral sense to a limit form defined on a subspace of $H^{1}(\Omega)$. This result is the basis of all the applications presented in the next section.

We begin with some useful definitions: 
Definition 3.1. Let $H$ be a vector space and $V$ be a linear subspace of $H$. Let $a: V \times V \rightarrow \mathbb{R}$ and $b: H \times H \rightarrow \mathbb{R}$ be bilinear forms. If $\lambda \in \mathbb{R}$, $u \in V \backslash\{0\}$ satisfy

$$
a(u, v)=\lambda b(u, v) \quad \text { for all } v \in V
$$

then we say that $\lambda$ is an eigenvalue of the pair $(a, b)$ and $u$ is an eigenvector of the pair $(a, b)$, corresponding to $\lambda$. The dimension of the span of all eigenvectors of $(a, b)$ corresponding to $\lambda$ is called the multiplicity of $\lambda$. If the set of eigenvalues of $(a, b)$ is countably infinite, contains a smallest element and if each eigenvalue has finite multiplicity then the repeated sequence of eigenvalues of $(a, b)$ is the uniquely determined nondecreasing sequence $\left(\lambda_{n}\right)_{n \in \mathbb{N}}$ which contains exactly the eigenvalues of $(a, b)$ and the number of occurrences of each eigenvalue in this sequence is equal to its multiplicity.

Given $a$ and $b$ as above define $R=R(a, b)$ to be the set of all pairs $(u, w) \in V \times H$ such that $a(u, v)=b(w, v)$ for all $v \in V$. We call $R$ the operator relation generated by the pair $(a, b)$. If $R$ is the graph of a map $A: D(A) \rightarrow H$, then this map is called the operator generated by the pair $(a, b)$.

The following properties are obvious:

Proposition 3.2. Let $H, V, a, b$ and $R$ be as in Definition 3.1. Then $R$ is a linear subspace of $V \times H$. Moreover, $(\lambda, u)$ is an eigenvalue-eigenvector pair for $(a, b)$ if and only if $\lambda \in \mathbb{R}, u \in V, u \neq 0$ and $(u, \lambda u) \in R$. Thus if $R$ is the graph of a map $A$, then $A$ is linear and $(\lambda, u)$ is an eigenvalueeigenvector pair for $(a, b)$ if and only if $(\lambda, u)$ is an eigenvalue-eigenvector pair for $A$.

The following proposition is well known:

Proposition 3.3. Let $V, H$ be two infinite-dimensional Hilbert spaces. Suppose $V \subset H$ with compact inclusion, and $V$ is dense in $H$. Let $\|\cdot\|$ and $|\cdot|$ denote the norms of $V$ and $H$ respectively, and $b$ be the inner product of $H$. Let $a: V \times V \rightarrow \mathbb{R}$ be a symmetric bilinear form on $V$. Assume that there are constants $d, C, \alpha \in \mathbb{R}, \alpha>0$, such that, for all $u, v \in V$,

$$
|a(u, v)| \leq C\|u\| \cdot\|v\|, \quad a(u, u) \geq \alpha\|u\|^{2}-d|u|^{2} .
$$

Then the set of eigenvalues of $(a, b)$ is countably infinite, it has a smallest element and each eigenvalue has finite multiplicity. Moreover, the operator relation generated by $(a, b)$ is the graph of a linear selfadjoint operator $A$ on $(H,\langle\cdot, \cdot\rangle)$ with compact resolvent.

For $\varepsilon \in] 0,1]$ define the bilinear forms $\widetilde{a}_{\varepsilon}: H^{1}\left(\Omega_{\varepsilon}\right) \times H^{1}\left(\Omega_{\varepsilon}\right) \rightarrow \mathbb{R}$ and $a_{\varepsilon}: H^{1}(\Omega) \times H^{1}(\Omega) \rightarrow \mathbb{R}$ by

$$
\tilde{a}_{\varepsilon}(u, v):=\int_{\Omega_{\varepsilon}} \nabla u(x) \cdot \nabla v(x) d x,
$$




$$
\begin{aligned}
a_{\varepsilon}(u, v):= & \int_{\Omega} J_{\varepsilon}(x)\left\langle S_{\varepsilon}(x)^{T} \nabla u(x), S_{\varepsilon}(x)^{T} \nabla v(x)\right\rangle d x \\
& +\frac{1}{\varepsilon^{2}} \int_{\Omega} J_{\varepsilon}(x)\langle P(x) \nabla u(x), P(x) \nabla v(x)\rangle d x .
\end{aligned}
$$

Moreover, for $\varepsilon \in[0,1]$ define the bilinear forms $\widetilde{b}_{\varepsilon}: L^{2}\left(\Omega_{\varepsilon}\right) \times L^{2}\left(\Omega_{\varepsilon}\right) \rightarrow \mathbb{R}$ and $b_{\varepsilon}: L^{2}(\Omega) \times L^{2}(\Omega) \rightarrow \mathbb{R}$ by

$$
\begin{aligned}
\widetilde{b}_{\varepsilon}(u, v) & :=\int_{\Omega_{\varepsilon}} u(x) v(x) d x, \\
b_{\varepsilon}(u, v) & :=\int_{\Omega} J_{\varepsilon}(x) u(x) v(x) d x .
\end{aligned}
$$

Note that by Theorem 2.4 there are constants $C, c \in] 0, \infty[$ such that

$$
c b_{\varepsilon}(u, u) \leq|u|_{L^{2}(\Omega)}^{2} \leq C b_{\varepsilon}(u, u) \quad \text { for } \varepsilon \in[0,1] \text { and } u \in L^{2}(\Omega) .
$$

It is clear that, for $\varepsilon \in] 0,1]$, the assignment $u \mapsto u \circ \Phi_{\varepsilon}$ restricts to linear isomorphisms $L^{2}\left(\Omega_{\varepsilon}\right) \rightarrow L^{2}(\Omega)$ and $H^{1}\left(\Omega_{\varepsilon}\right) \rightarrow H^{1}(\Omega)$. Using the changeof-variables formula and Theorem 2.4 we see that, for $\varepsilon \in] 0,1]$,

$$
a_{\varepsilon}\left(u \circ \Phi_{\varepsilon}, v \circ \Phi_{\varepsilon}\right)=\varepsilon^{-(l-k) / 2} \widetilde{a}_{\varepsilon}(u, v) \quad \text { for all } u, v \in H^{1}\left(\Omega_{\varepsilon}\right) .
$$

Moreover,

$$
b_{\varepsilon}\left(u \circ \Phi_{\varepsilon}, v \circ \Phi_{\varepsilon}\right)=\varepsilon^{-(l-k) / 2} \widetilde{b}_{\varepsilon}(u, v) \quad \text { for all } u, v \in L^{2}\left(\Omega_{\varepsilon}\right) .
$$

We thus obtain the following

Proposition 3.4. The (linear) operators $\widetilde{A}_{\varepsilon}$ (resp. $\left.A_{\varepsilon}\right)$ defined by $\left(\widetilde{a}_{\varepsilon}, \widetilde{b}_{\varepsilon}\right)$ (resp. $\left.\left(a_{\varepsilon}, b_{\varepsilon}\right)\right)$ have the following properties:

(1) $u \in \widetilde{D}\left(A_{\varepsilon}\right)$ if and only if $u \circ \Phi_{\varepsilon} \in D\left(A_{\varepsilon}\right)$;

(2) $A_{\varepsilon}\left(u \circ \Phi_{\varepsilon}\right)=\left(\widetilde{A}_{\varepsilon} u\right) \circ \Phi_{\varepsilon}$ for $u \in D\left(\widetilde{A}_{\varepsilon}\right)$.

Notice that if $u \in H^{1}(\Omega)$, then

$$
\lim _{\varepsilon \rightarrow 0} a_{\varepsilon}(u, u)= \begin{cases}\int_{\Omega} J_{0}(x)\left\langle S_{0}(x)^{T} \nabla u(x), S_{0}(x)^{T} \nabla v(x)\right\rangle d x \\ & \text { if } P(x) \nabla u(x)=0 \text { a.e. } \\ \infty & \text { otherwise. }\end{cases}
$$

Define

$$
H_{\mathrm{s}}^{1}(\Omega):=\left\{u \in H^{1}(\Omega) \mid P(x) \nabla u(x)=0 \text { a.e. }\right\} .
$$

Note that $H_{\mathrm{S}}^{1}(\Omega)$ is a closed linear subspace of the Hilbert space $H^{1}(\Omega)$.

Proposition 3.5. The space $H_{\mathrm{s}}^{1}(\Omega)$ is infinite-dimensional.

Proof. Since $\Omega$ is open and nonempty and $\phi: \mathcal{U} \rightarrow \mathcal{M}$ is open by Theorem 2.4, it follows that $V:=\phi(\mathcal{U})$ is open and nonempty in $\mathcal{M}$. Let $C_{\mathrm{c}}^{1}(V)$ be the linear space of all real-valued $C^{1}$-functions on $\mathcal{M}$ with compact support contained in $V$. For every $u \in C_{\mathrm{c}}^{1}(V)$ the function $u \circ \phi$ is bounded 
and has continuous and bounded derivatives. Thus $u \circ \phi \in H^{1}(\Omega)$. Moreover, for $x \in \Omega$ the vector $\nu=\nu(x):=P(x) \nabla(u \circ \phi)(x)$ is orthogonal to $T_{\phi(x)}(\mathcal{M})$. Thus

$$
\langle\nu, \nu\rangle=\langle P(x) \nabla(u \circ \phi)(x), \nu\rangle=\langle\nabla(u \circ \phi)(x), \nu\rangle=D(u \circ \phi)(x) \nu=0
$$

since $D \phi(x) \nu=0$. Hence $\nu(x)=0$ on $\Omega$, i.e. $u \circ \phi \in H_{\mathrm{s}}^{1}(\Omega)$. Define the map $\Gamma: C_{\mathrm{c}}^{1}(V) \rightarrow H_{\mathrm{s}}^{1}(\Omega)$ by $u \mapsto u \circ \phi$. Clearly, $\Gamma$ is injective. Since $C_{\mathrm{c}}^{1}(V)$ has infinite dimension, so does $H_{\mathrm{s}}^{1}(\Omega)$.

Now define the "limit" bilinear form $a_{0}: H_{\mathrm{s}}^{1}(\Omega) \times H_{\mathrm{s}}^{1}(\Omega) \rightarrow \mathbb{R}$ by

$$
a_{0}(u, v):=\int_{\Omega} J_{0}(x)\left\langle S_{0}(x)^{T} \nabla u(x), S_{0}(x)^{T} \nabla v(x)\right\rangle d x .
$$

Finally, let $L_{\mathrm{s}}^{2}(\Omega)$ be the closure of $H_{\mathrm{s}}^{1}(\Omega)$ in $L^{2}(\Omega)$. We will denote by $A_{0}$ the operator generated by the pair $\left(a_{0},\left.b_{0}\right|_{L_{\mathrm{s}}^{2}(\Omega) \times L_{\mathrm{s}}^{2}(\Omega)}\right)$.

For $\varepsilon \in[0,1]$ and $u \in L^{2}(\Omega)$ set

$$
|u|_{\varepsilon}:=b_{\varepsilon}(u, u)^{1 / 2} \text {. }
$$

For $\varepsilon \in] 0,1]$ and $u \in H^{1}(\Omega)$ set

$$
\|u\|_{\varepsilon}:=\left(a_{\varepsilon}(u, u)+b_{\varepsilon}(u, u)\right)^{1 / 2} .
$$

Finally, for $\varepsilon=0$ and $u \in H_{\mathrm{S}}^{1}(\Omega)$ set

$$
\|u\|_{0}:=\left(a_{0}(u, u)+b_{0}(u, u)\right)^{1 / 2} .
$$

We need the following propositions.

Proposition 3.6. For every compact set $K \subset \mathcal{U}$ and $\delta \in] 0,1[$ there exists an $\bar{\varepsilon} \in] 0,1]$ such that, for all $\varepsilon \in] 0, \bar{\varepsilon}], x \in K$ and $h \in T_{\phi(x)} \mathcal{M}$,

$$
\begin{aligned}
(1-\delta)\left\langle S_{0}(x)^{T} h, S_{0}(x)^{T} h\right\rangle \leq_{(1)}\left\langle S_{\varepsilon}(x)^{T} h, S_{\varepsilon}(x)^{T} h\right\rangle \\
\quad \leq_{(2)}(1+\delta)\left\langle S_{0}(x)^{T} h, S_{0}(x)^{T} h\right\rangle .
\end{aligned}
$$

Proof. Assume by contradiction that $\leq_{(1)}$ is false. Then there exists a $\delta \in] 0,1\left[\right.$, and for all $m \in \mathbb{N}$ there exist $x_{m} \in K, \varepsilon_{m} \in \mathbb{R}, 0<\varepsilon_{m}<1 / m$ and $h_{m} \in T_{\phi\left(x_{m}\right)} \mathcal{M}$, such that

$$
(1-\delta)\left\langle S_{0}\left(x_{m}\right)^{T} h_{m}, S_{0}\left(x_{m}\right)^{T} h_{m}\right\rangle \geq\left\langle S_{\varepsilon_{m}}\left(x_{m}\right)^{T} h_{m}, S_{\varepsilon_{m}}\left(x_{m}\right)^{T} h_{m}\right\rangle .
$$

We can assume without loss of generality that $\left|h_{m}\right|=1$ for all $m$. Then, up to a subsequence, $x_{m} \rightarrow x$ and $h_{m} \rightarrow h$ as $m \rightarrow \infty$, where $x \in K$ and $|h|=1$. It is not difficult to see that $h \in T_{\phi(x)} \mathcal{M}$. Letting $m \rightarrow \infty$ in (47) and applying Theorem 2.4 we obtain

$$
(1-\delta)\left\langle S_{0}(x)^{T} h, S_{0}(x)^{T} h\right\rangle \geq\left\langle S_{0}(x)^{T} h, S_{0}(x)^{T} h\right\rangle .
$$

It follows that $S_{0}(x)^{T} h=0$, so $h=0$ by Theorem 2.4, a contradiction. The proof of $\leq_{(2)}$ is analogous. 
Proposition 3.7. For every compact set $K \subset \mathcal{U}$ there is a constant $\gamma=\gamma(K) \in] 0, \infty\left[\right.$ such that, for all $x \in K, \varepsilon \in[0,1]$ and $h \in T_{\phi(x)} \mathcal{M}$,

$$
\left\langle S_{\varepsilon}(x)^{T} h, S_{\varepsilon}(x)^{T} h\right\rangle \geq \gamma|h|^{2} .
$$

Proof. Assume by contradiction that the proposition is false. Then for all $m \in \mathbb{N}$ there exist $x_{m} \in K, \varepsilon_{m} \in[0,1]$ and $h_{m} \in T_{\phi\left(x_{m}\right)} \mathcal{M}$ such that

$$
\frac{1}{m}\left|h_{m}\right|^{2} \geq\left\langle S_{\varepsilon_{m}}\left(x_{m}\right)^{T} h_{m}, S_{\varepsilon_{m}}\left(x_{m}\right)^{T} h_{m}\right\rangle
$$

We can assume that $\left|h_{m}\right|=1$ for all $m$. So, up to a subsequence, $x_{m} \rightarrow x$, $\varepsilon_{m} \rightarrow \varepsilon$ and $h_{m} \rightarrow h$ as $m \rightarrow \infty$, where $x \in K, \varepsilon \in[0,1]$ and $|h|=1$. It is not difficult to see that $h \in T_{\phi(x)} \mathcal{M}$. Letting $m \rightarrow \infty$ in (49) and applying Theorem 2.4 we obtain

$$
\left\langle S_{\varepsilon}(x)^{T} h, S_{\varepsilon}(x)^{T} h\right\rangle=0 .
$$

It follows that $S_{0}(x)^{T} h=0$, so $h=0$ by Theorem 2.4, a contradiction.

As a consequence of Propositions 3.6 and 3.7, we have:

Proposition 3.8. For every $\delta \in] 0,1[$ there exists an $\bar{\varepsilon} \in] 0,1]$ such that

$$
(1-\delta) b_{0}(u, u) \leq b_{\varepsilon}(u, u) \leq(1+\delta) b_{0}(u, u)
$$

for all $u \in L^{2}$ and $\left.\left.\varepsilon \in\right] 0, \bar{\varepsilon}\right]$, and

$$
(1-\delta) a_{0}(u, u) \leq a_{\varepsilon}(u, u) \leq(1+\delta) a_{0}(u, u)
$$

for all $u \in H_{\mathrm{S}}^{1}(\Omega)$ and $\left.\varepsilon \in\right] 0, \bar{\varepsilon}[$.

Furthermore, whenever $u, v \in L^{2}$, then

$$
b_{\varepsilon}(u, v) \rightarrow b_{0}(u, v) \quad \text { as } \varepsilon \rightarrow 0 .
$$

Moreover, on $H_{\mathrm{s}}^{1}(\Omega)$ the norms $\|\cdot\|_{\varepsilon}$ and $\|\cdot\|_{0}$ are equivalent, with equivalence constants independent of $\varepsilon \in] 0,1]$, and

$$
a_{\varepsilon}(u, u) \rightarrow a_{0}(u, u) \quad \text { as } \varepsilon \rightarrow 0
$$

for all $u \in H_{\mathrm{S}}^{1}(\Omega)$. Finally, there exists a $\gamma>0$ such that

$$
\left.\left.\gamma|u|_{H^{1}} \leq\|u\|_{\varepsilon} \quad \text { for all } \varepsilon \in\right] 0,1\right] \text { and } u \in H^{1} .
$$

In the rest of this section we will present a basic spectral convergence result, which generalizes [21, Theorem 3.3] and has far-reaching implications concerning the dynamics of both reaction-diffusion equations and damped wave equations on thin domains.

Since we are working on a fixed domain $\Omega$, we can write for short $L^{2}:=$ $L^{2}(\Omega), H^{1}:=H^{1}(\Omega), L_{\mathrm{s}}^{2}:=L_{\mathrm{s}}^{2}(\Omega)$ and $H_{\mathrm{s}}^{1}:=H_{\mathrm{s}}^{1}(\Omega)$. By $(43)$ the norms $|\cdot|_{\varepsilon}, \varepsilon \in[0,1]$, are all equivalent to the usual norm on $L^{2}$, with equivalence constants independent of $\varepsilon$.

For $\varepsilon \in] 0,1]$ the norm $\|\cdot\|_{\varepsilon}$ is equivalent to the usual norm on $H^{1}$ and so $\left(H^{1},\|\cdot\|_{\varepsilon}\right)$ is densely and compactly imbedded in $\left(L^{2},|\cdot|_{\varepsilon}\right)$. 
Consequently, well known results (cf. [21, Proposition 2.2]) imply that, for all $\varepsilon \in] 0,1]$, there exists a sequence $\left(\lambda_{j}^{\varepsilon}, w_{j}^{\varepsilon}\right)_{j \in \mathbb{N}}$ of eigenvalue-eigenvector pairs for $\left(a_{\varepsilon}, b_{\varepsilon}\right)$ such that $\lambda_{1}^{\varepsilon} \leq \lambda_{2}^{\varepsilon} \leq \lambda_{3}^{\varepsilon} \leq \ldots$ and $\left(w_{j}^{\varepsilon}\right)_{j \in \mathbb{N}}$ is a complete $b_{\varepsilon}$-orthonormal system in $L^{2}$.

Similarly, $H_{\mathrm{s}}^{1}$ is densely and compactly imbedded in $L_{\mathrm{s}}^{2}$. Since $H_{\mathrm{s}}^{1}$ has infinite dimension by Proposition 3.5, [21, Proposition 2.2] again implies that there exists a sequence $\left(\lambda_{j}^{0}, w_{j}^{0}\right)_{j \in \mathbb{N}}$ of eigenvalue-eigenvector pairs for $\left(a_{0}, b_{0}\right)$ such that $\lambda_{1}^{0} \leq \lambda_{2}^{0} \leq \lambda_{3}^{0} \leq \ldots$ and $\left(w_{j}^{0}\right)_{j \in \mathbb{N}}$ is a complete $b_{0}$-orthonormal system in $L_{\mathrm{s}}^{2}$.

We need the following

Lemma 3.9. Let $\left(\varepsilon_{m}\right)_{m \in \mathbb{N}}$ be a sequence in $\left.] 0,1\right]$ converging to zero. Let $\left(u_{m}\right)_{m \in \mathbb{N}}$ be a sequence in $H^{1}$ and assume there exists $u \in H_{\mathrm{s}}^{1}$ such that $\left\|u_{m}-u\right\|_{\varepsilon_{m}} \rightarrow 0$ as $m \rightarrow \infty$. Then

$$
\left|a_{\varepsilon_{m}}\left(u_{m}, u_{m}\right)-a_{0}(u, u)\right| \rightarrow 0 \quad \text { as } m \rightarrow \infty .
$$

Proof. We begin by computing

$$
\begin{aligned}
\leq & \left|a_{\varepsilon_{m}}\left(u_{m}, u_{m}\right)+b_{\varepsilon_{m}}\left(u_{m}, u_{m}\right)-a_{\varepsilon_{m}}(u, u)-b_{\varepsilon_{m}}(u, u)\right| \\
& +\left|a_{\varepsilon_{m}}(u, u)-a_{0}(u, u)\right|+\left|b_{\varepsilon_{m}}\left(u_{m}, u_{m}\right)-b_{\varepsilon_{m}}(u, u)\right| \\
= & ||\left|u_{m}\left\|_{\varepsilon_{m}}^{2}-\right\| u \|_{\varepsilon_{m}}^{2}\right|+\left|a_{\varepsilon_{m}}(u, u)-a_{0}(u, u)\right|+\left|b_{\varepsilon_{m}}\left(u_{m}, u_{m}\right)-b_{\varepsilon_{m}}(u, u)\right| .
\end{aligned}
$$

Since $\|\cdot\|_{\varepsilon_{m}}$ and $|\cdot|_{\varepsilon_{m}}$ are norms we have

$$
\begin{aligned}
& \left|\left\|u_{m}\right\|_{\varepsilon_{m}}-\|u\|_{\varepsilon_{m}}\right| \leq\left\|u_{m}-u\right\|_{\varepsilon_{m}} \rightarrow 0 \quad \text { as } m \rightarrow \infty, \\
& \left.|| u_{m}\right|_{\varepsilon_{m}}-|u|_{\varepsilon_{m}}|\leq| u_{m}-\left.u\right|_{\varepsilon_{m}} \rightarrow 0 \quad \text { as } m \rightarrow \infty \text {. }
\end{aligned}
$$

However, $\|u\|_{\varepsilon_{m}} \rightarrow\|u\|_{0}$ and $|u|_{\varepsilon_{m}} \rightarrow|u|_{0}$ as $m \rightarrow \infty$ by (52) and (53). Thus also $\left\|u_{m}\right\|_{\varepsilon_{m}} \rightarrow\|u\|_{0}$ and hence

$$
\left|\left\|u_{m}\right\|_{\varepsilon_{m}}^{2}-\|u\|_{\varepsilon_{m}}^{2}\right| \rightarrow 0
$$

In the same way, also $\left|u_{m}\right|_{\varepsilon_{m}} \rightarrow|u|_{0}$ and hence

$$
\left|b_{\varepsilon_{m}}\left(u_{m}, u_{m}\right)-b_{\varepsilon_{m}}(u, u)\right|=\left.|| u_{m}\right|_{\varepsilon_{m}} ^{2}-|u|_{\varepsilon_{m}}^{2} \mid \rightarrow 0 .
$$

Finally, (57), (58) and (53) imply that (55) is satisfied.

With these preliminaries, we have the following

Theorem 3.10. For $\varepsilon \in] 0,1]$ let $\lambda_{1}^{\varepsilon} \leq \lambda_{2}^{\varepsilon} \leq \lambda_{3}^{\varepsilon} \leq \ldots$ be the repeated sequence of eigenvalues of the pair $\left(a_{\varepsilon}, b_{\varepsilon}\right)$ and $w_{1}^{\varepsilon}, w_{2}^{\varepsilon}, w_{3}^{\varepsilon}, \ldots$ be a corresponding complete $\left(L^{2}, b_{\varepsilon}\right)$-orthonormal sequence of eigenvectors. Moreover, let $\lambda_{1}^{0} \leq \lambda_{2}^{0} \leq \lambda_{3}^{0} \leq \ldots$ be the repeated sequence of eigenvalues of $\left(a_{0}, b_{0}\right)$. Then: 
(1) For every $j \in \mathbb{N}$,

$$
\lambda_{j}^{0}=\lim _{\varepsilon \rightarrow 0^{+}} \lambda_{j}^{\varepsilon}
$$

(2) Let $\left(\varepsilon_{n}\right)_{n \in \mathbb{N}}$ be a sequence of positive numbers converging to 0 . Then there is a subsequence of $\left(\varepsilon_{n}\right)_{n \in \mathbb{N}}$, again denoted by $\left(\varepsilon_{n}\right)_{n \in \mathbb{N}}$, and a complete $\left(L_{\mathrm{s}}^{2}, b_{0}\right)$-orthonormal system $\left(w_{j}^{0}\right)_{j \in \mathbb{N}}$ of eigenvectors of $\left(a_{0}, b_{0}\right)$ corresponding to $\left(\lambda_{j}^{0}\right)_{j \in \mathbb{N}}$ such that, for every $j \in \mathbb{N}$,

$$
\left\|w_{j}^{\varepsilon_{n}}-w_{j}^{0}\right\|_{\varepsilon_{n}} \rightarrow 0 \quad \text { as } n \rightarrow \infty .
$$

Proof. Let $j \in \mathbb{N}$. By [21, Proposition 2.2],

$$
\lambda_{j}^{\varepsilon}=\min _{E \in \mathcal{F}_{j}} \max _{u \in E \backslash\{0\}} \frac{a_{\varepsilon}(u, u)}{b_{\varepsilon}(u, u)},
$$

where $\mathcal{F}_{j}$ is the set of all $j$-dimensional linear subspaces of $H^{1}$. Since $H_{\mathrm{s}}^{1} \subset$ $H^{1}$, by (50) and (51) for every $\left.\delta \in\right] 0,1[$ there exists an $\left.\bar{\varepsilon}(\delta) \in] 0,1\right]$ such that, for all $\varepsilon \in] 0, \bar{\varepsilon}]$,

$$
0 \leq \lambda_{j}^{\varepsilon}=\min _{E \in \mathcal{F}_{j}} \max _{u \in E \backslash\{0\}} \frac{a_{\varepsilon}(u, u)}{b_{\varepsilon}(u, u)} \leq \frac{1+\delta}{1-\delta} \min _{E \in \mathcal{F}_{\text {s } j}} \max _{u \in E \backslash\{0\}} \frac{a_{0}(u, u)}{b_{0}(u, u)}=\frac{1+\delta}{1-\delta} \lambda_{j}^{0}
$$

where $\mathcal{F}_{\mathrm{s} j}$ is the set of all $j$-dimensional linear subspaces of $H_{\mathrm{s}}^{1}$. Thus the set $\left\{\lambda_{j}^{\varepsilon} \mid \varepsilon \in\right] 0,1[\}$ is bounded in $\mathbb{R}$. Now let $\left(\varepsilon_{m}\right)_{m \in \mathbb{N}}$ be a sequence of positive numbers converging to zero. It follows that there is a subsequence of $\left(\varepsilon_{m}\right)_{m \in \mathbb{N}}$ (again denoted by $\left(\varepsilon_{m}\right)_{m \in \mathbb{N}}$ ) and a number $\mu_{j}$ such that

$$
\mu_{j}=\lim _{m \rightarrow \infty} \lambda_{j}^{\varepsilon_{m}} .
$$

Let $\left(\delta_{m}\right)_{m \in \mathbb{N}}$ be any sequence of positive numbers converging to zero. We can assume that $\varepsilon_{m} \leq \bar{\varepsilon}\left(\delta_{m}\right)$ for all $m$. Note that $\mu_{j} \leq \lambda_{j}^{0}$.

Now, for $j$ fixed and all $m \in \mathbb{N}$, we have

$$
\begin{aligned}
a_{\varepsilon_{m}}\left(w_{j}^{\varepsilon_{m}}, w_{j}^{\varepsilon_{m}}\right) & =\lambda_{j}^{\varepsilon_{m}}, \\
a_{\varepsilon_{m}}\left(w_{j}^{\varepsilon_{m}}, w\right) & =\lambda_{j}^{\varepsilon_{m}} b_{\varepsilon_{m}}\left(w_{j}^{\varepsilon_{m}}, w\right) \quad \text { for all } w \in H^{1} .
\end{aligned}
$$

Hence

$$
\begin{aligned}
\gamma\left|w_{j}^{\varepsilon_{m}}\right|_{H^{1}}^{2} & \leq a_{\varepsilon_{m}}\left(w_{j}^{\varepsilon_{m}}, w_{j}^{\varepsilon_{m}}\right)+b_{\varepsilon_{m}}\left(w_{j}^{\varepsilon_{m}}, w_{j}^{\varepsilon_{m}}\right) \\
& =\lambda_{j}^{\varepsilon_{m}}+1 \leq \frac{1+\delta_{m}}{1-\delta_{m}} \lambda_{j}^{0}+1 .
\end{aligned}
$$

It follows that there exists a subsequence of $\left(\varepsilon_{m}\right)_{m \in \mathbb{N}}$ (again denoted by $\left.\left(\varepsilon_{m}\right)_{m \in \mathbb{N}}\right)$ and a function $w_{j} \in H^{1}(\Omega)$ such that

$$
w_{j}^{\varepsilon_{m}} \rightarrow w_{j} \quad \text { in } H^{1}(\Omega)
$$

as $m \rightarrow \infty$. Since $\Omega$ has Lipschitz boundary, the space $H^{1}(\Omega)$ is compactly imbedded into $L^{2}(\Omega)$, so

$$
w_{j}^{\varepsilon_{m}} \rightarrow w_{j} \quad \text { in } L^{2}(\Omega) .
$$


For all $m$ we have

$$
\left(1-\delta_{m}\right) b_{0}\left(w_{j}^{\varepsilon_{m}}, w_{j}^{\varepsilon_{m}}\right) \leq b_{\varepsilon_{m}}\left(w_{j}^{\varepsilon_{m}}, w_{j}^{\varepsilon_{m}}\right)=1 \leq\left(1+\delta_{m}\right) b_{0}\left(w_{j}^{\varepsilon_{m}}, w_{j}^{\varepsilon_{m}}\right),
$$

so, passing to the limit, we obtain $b_{0}\left(w_{j}, w_{j}\right)=1$.

Next we show that $w_{j} \in H_{\mathrm{s}}^{1}$. Observe that

$$
\begin{aligned}
& \frac{1}{\varepsilon_{m}^{2}} \int_{\Omega} J_{\varepsilon}(x)\left\langle P(x) \nabla w_{j}^{\varepsilon_{m}}(x), P(x) \nabla w_{j}^{\varepsilon_{m}}(x)\right\rangle d x \\
& \quad \leq a_{\varepsilon_{m}}\left(w_{j}^{\varepsilon_{m}}(x), w_{j}^{\varepsilon_{m}}(x)\right)=\lambda_{j}^{\varepsilon_{m}} \leq \frac{1+\delta_{m}}{1-\delta_{m}} \lambda_{j}^{0},
\end{aligned}
$$

which implies that

$$
\int_{\Omega} J_{\varepsilon_{m}}(x)\left\langle P(x) \nabla w_{j}^{\varepsilon_{m}}(x), P(x) \nabla w_{j}^{\varepsilon_{m}}(x)\right\rangle d x \rightarrow 0 \quad \text { as } m \rightarrow \infty,
$$

so $P(\cdot) \nabla w_{j}^{\varepsilon_{m}} \rightarrow 0$ in $L^{2}\left(\Omega, \mathbb{R}^{l}\right)$. However, since $\nabla w_{j}^{\varepsilon_{m}} \rightarrow \nabla w_{j}$ in $L^{2}\left(\Omega, \mathbb{R}^{l}\right)$ and the map $x \mapsto P(x)$ is continuous from $\bar{\Omega}$ to $\mathcal{L}\left(\mathbb{R}^{l}, \mathbb{R}^{l}\right)$, we deduce that $P(\cdot) \nabla w_{j}^{\varepsilon_{m}} \rightarrow P(\cdot) \nabla w_{j}$ in $L^{2}\left(\Omega, \mathbb{R}^{l}\right)$ as $m \rightarrow \infty$. Thus $P(x) \nabla w_{j}(x)=0$ a.e. in $\Omega$, i.e. $w_{j} \in H_{\mathrm{s}}^{1}$.

Now we prove that $\left(\mu_{j}, w_{j}\right)$ is an eigenvalue-eigenvector pair for $\left(a_{0}, b_{0}\right)$. Let $w \in H_{\mathrm{s}}^{1}$. Since $P(x) \nabla w(x)=0$ almost everywhere, we have

$$
\begin{aligned}
& \int_{\Omega} J_{\varepsilon_{m}}(x)\left\langle S_{\varepsilon_{m}}(x)^{T} \nabla w_{j}^{\varepsilon_{m}}(x), S_{\varepsilon_{m}}(x)^{T} \nabla w(x)\right\rangle d x \\
& \quad=a_{\varepsilon_{m}}\left(w_{j}^{\varepsilon_{m}}, w\right)=\lambda_{j}^{\varepsilon_{m}} b_{\varepsilon_{m}}\left(w_{j}^{\varepsilon_{m}}, w\right)=\lambda_{j}^{\varepsilon_{m}} \int_{\Omega} J_{\varepsilon_{m}}(x) w_{j}^{\varepsilon_{m}}(x) w(x) d x .
\end{aligned}
$$

Since $w_{j}^{\varepsilon_{m}} \rightarrow w_{j}$ in $H^{1}(\Omega), S_{\varepsilon_{m}}(x)^{T} \rightarrow S_{0}(x)^{T}$ in $\mathcal{L}\left(\mathbb{R}^{l}, \mathbb{R}^{l}\right)$ uniformly on $\bar{\Omega}, J_{\varepsilon_{m}}(x) \rightarrow J_{0}(x)$ in $\mathbb{R}$ uniformly on $\bar{\Omega}, \lambda_{j}^{\varepsilon_{m}} \rightarrow \mu_{j}$ and $w_{j}^{\varepsilon_{m}} \rightarrow w_{j}$ in $L^{2}(\Omega)$ as $m \rightarrow \infty$, we conclude that

$$
a_{0}\left(w_{j}, w\right)=\int_{\Omega} J_{0}(x)\left\langle S_{0}(x)^{T} \nabla w_{j}(x), S_{0}(x)^{T} \nabla w(x)\right\rangle d x=\mu_{j} b_{0}\left(w_{j}, w\right),
$$

i.e. $\left(\mu_{j}, w_{j}\right)$ is an eigenvalue-eigenvector pair for $\left(a_{0}, b_{0}\right)$.

Now we prove that $\left\|w_{j}^{\varepsilon_{m}}-w_{j}\right\|_{\varepsilon_{m}} \rightarrow 0$ as $m \rightarrow \infty$. We have

$$
\begin{aligned}
& \left\|w_{j}^{\varepsilon_{m}}-w_{j}\right\|_{\varepsilon_{m}}^{2}=a_{\varepsilon_{m}}\left(w_{j}^{\varepsilon_{m}}-w_{j}, w_{j}^{\varepsilon_{m}}-w_{j}\right)+b_{\varepsilon_{m}}\left(w_{j}^{\varepsilon_{m}}-w_{j}, w_{j}^{\varepsilon_{m}}-w_{j}\right) \\
= & \lambda_{j}^{\varepsilon_{m}}-2 a_{\varepsilon_{m}}\left(w_{j}^{\varepsilon_{m}}, w_{j}\right)+a_{\varepsilon_{m}}\left(w_{j}, w_{j}\right)+b_{\varepsilon_{m}}\left(w_{j}^{\varepsilon_{m}}-w_{j}, w_{j}^{\varepsilon_{m}}-w_{j}\right) \\
= & \lambda_{j}^{\varepsilon_{m}}-2 \lambda_{j}^{\varepsilon_{m}} b_{\varepsilon_{m}}\left(w_{j}^{\varepsilon_{m}}, w_{j}\right)+a_{\varepsilon_{m}}\left(w_{j}, w_{j}\right)+b_{\varepsilon_{m}}\left(w_{j}^{\varepsilon_{m}}-w_{j}, w_{j}^{\varepsilon_{m}}-w_{j}\right) .
\end{aligned}
$$

Observe that $b_{\varepsilon_{m}}\left(w_{j}^{\varepsilon_{m}}, w_{j}\right) \rightarrow b_{0}\left(w_{j}, w_{j}\right)=1, \lambda_{j}^{\varepsilon_{n}} \rightarrow \mu_{j}$ and $a_{\varepsilon_{m}}\left(w_{j}, w_{j}\right) \rightarrow$ $a_{0}\left(w_{j}, w_{j}\right)=\mu_{j}$ as $m \rightarrow \infty$. Moreover,

$$
b_{\varepsilon_{m}}\left(w_{j}^{\varepsilon_{m}}-w_{j}, w_{j}^{\varepsilon_{m}}-w_{j}\right) \leq\left(1+\delta_{m}\right) b_{0}\left(w_{j}^{\varepsilon_{m}}-w_{j}, w_{j}^{\varepsilon_{m}}-w_{j}\right) \rightarrow 0
$$


as $m \rightarrow \infty$. These properties together imply that $\left\|w_{j}^{\varepsilon_{m}}-w_{j}\right\|_{\varepsilon_{m}} \rightarrow 0$ as $m \rightarrow \infty$. In particular, by (54), $w_{j}^{\varepsilon_{m}} \rightarrow w_{j}$ in $H^{1}$ as $m \rightarrow \infty$.

By the Cantor diagonal procedure, given a sequence $\left(\varepsilon_{m}\right)_{m \in \mathbb{N}}$ of positive numbers converging to zero, we can find a subsequence (again denoted by $\left.\left(\varepsilon_{m}\right)_{m \in \mathbb{N}}\right)$ and a family $\left(w_{j}\right)_{j \in \mathbb{N}}$ of functions in $H_{\mathrm{s}}^{1}$ with the following properties:

(1) for every $j \in \mathbb{N},\left(\mu_{j}, w_{j}\right)$ is an eigenvalue-eigenvector pair for $\left(a_{0}, b_{0}\right)$;

(2) $b_{0}\left(w_{j}, w_{j}\right)=1$

(3) $\left\|w_{j}^{\varepsilon_{m}}-w_{j}\right\|_{\varepsilon_{m}} \rightarrow 0$ as $m \rightarrow \infty$.

In order to complete the proof, we have to show the following:

(1) the family $\left(w_{j}\right)_{j \in \mathbb{N}}$ is a complete $b_{0}$-orthonormal system in $L_{\mathrm{s}}^{2}$;

(2) $\mu_{j}=\lambda_{j}^{0}$ for all $j \in \mathbb{N}$.

First we show that $\left(w_{j}\right)_{j \in \mathbb{N}}$ is a $b_{0}$-orthonormal system in $L_{\mathrm{s}}^{2}$. Let $j_{1}, j_{2}$ $\in \mathbb{N}, j_{1} \neq j_{2}$. Then

$$
\int_{\Omega} J_{\varepsilon_{m}}(x) w_{j_{1}}^{\varepsilon_{m}}(x) w_{j_{2}}^{\varepsilon_{m}}(x) d x=0 \quad \text { for all } m \in \mathbb{N} .
$$

Since $w_{j}^{\varepsilon_{m}} \rightarrow w_{j}$ in $L^{2}$ as $m \rightarrow \infty$ for all $j \in \mathbb{N}$ and $J_{\varepsilon_{m}} \rightarrow J_{0}$ uniformly on $\bar{\Omega}$, it follows that

$$
\int_{\Omega} J_{0}(x) w_{j_{1}}(x) w_{j_{2}}(x) d x=0
$$

that is, $w_{j_{1}}$ and $w_{j_{2}}$ are $b_{0}$-orthogonal. By [21, Proposition 2.2], the proof will be complete if we show that, for every $j \in \mathbb{N}$,

$$
\begin{aligned}
\mu_{j}=a_{0}\left(w_{j}, w_{j}\right)=\min \left\{a_{0}(w, w) \mid\right. & w \in H_{\mathrm{s}}^{1}(\Omega), b_{0}(w, w)=1, \\
& \left.b_{0}\left(w, w_{h}\right)=0 \text { for } h=1, \ldots, j-1\right\} .
\end{aligned}
$$

Fix $j \in \mathbb{N}$, and let $w \in H_{\mathrm{s}}^{1}$ with $b_{0}(w, w)=1$ be such that $b_{0}\left(w, w_{h}\right)=0$ for $h=1, \ldots, j-1$. For $m \in \mathbb{N}$, define

$$
v_{m}:=w-\sum_{h=1}^{j-1} b_{\varepsilon_{m}}\left(w, w_{h}^{\varepsilon_{m}}\right) w_{h}^{\varepsilon_{m}} .
$$

Then obviously $b_{\varepsilon_{m}}\left(v_{m}, w_{h}^{\varepsilon_{m}}\right)=0$ for $h=1, \ldots, j-1$.

We claim that $\left\|v_{m}-w\right\|_{\varepsilon_{m}} \rightarrow 0$ as $m \rightarrow \infty$. In fact,

$$
\begin{aligned}
& \quad\left\|v_{n}-w\right\|_{\varepsilon_{m}}^{2}=\left\|\sum_{h=1}^{j-1} b_{\varepsilon_{m}}\left(w, w_{h}^{\varepsilon_{m}}\right) w_{h}^{\varepsilon_{m}}\right\|_{\varepsilon_{m}}^{2} \\
& =a_{\varepsilon_{m}}\left(\sum_{h=1}^{j-1} b_{\varepsilon_{m}}\left(w, w_{h}^{\varepsilon_{m}}\right) w_{h}^{\varepsilon_{m}}, \sum_{h=1}^{j-1} b\left(w, w_{h}^{\varepsilon_{m}}\right) w_{h}^{\varepsilon_{m}}\right)+\left|\sum_{h=1}^{j-1} b_{\varepsilon_{m}}\left(w, w_{h}^{\varepsilon_{n}}\right) w_{h}^{\varepsilon_{n}}\right|_{\varepsilon_{m}}^{2}
\end{aligned}
$$




$$
\begin{aligned}
& =\sum_{h, h^{\prime}=1}^{j-1} b_{\varepsilon_{m}}\left(w, w_{h}^{\varepsilon_{m}}\right) b_{\varepsilon_{m}}\left(w, w_{h^{\prime}}^{\varepsilon_{m}}\right) a_{\varepsilon_{m}}\left(w_{h}^{\varepsilon_{m}}, w_{h^{\prime}}^{\varepsilon_{m}}\right)+\sum_{h=1}^{j-1}\left|b_{\varepsilon_{m}}\left(w, w_{h}^{\varepsilon_{m}}\right)\right|^{2} \\
& =\sum_{h, h^{\prime}=1}^{j-1} b_{\varepsilon_{m}}\left(w, w_{h}^{\varepsilon_{m}}\right) b_{\varepsilon_{m}}\left(w, w_{h^{\prime}}^{\varepsilon_{m}}\right) \lambda_{h}^{\varepsilon_{m}} b_{\varepsilon_{m}}\left(w_{h}^{\varepsilon_{m}}, w_{h^{\prime}}^{\varepsilon_{m}}\right)+\sum_{h=1}^{j-1}\left|b_{\varepsilon_{m}}\left(w, w_{h}^{\varepsilon_{m}}\right)\right|^{2} .
\end{aligned}
$$

Since $w_{h}^{\varepsilon_{m}} \rightarrow w_{h}$ in $L^{2}$ as $m \rightarrow \infty$ for every $h=1, \ldots, j-1$, and $J_{\varepsilon_{m}} \rightarrow J_{0}$ uniformly on $\bar{\Omega}$ as well as $b_{0}\left(w, w_{h}\right)=0$ for $h=1, \ldots, j-1$, it follows that $\left\|v_{m}-w\right\|_{\varepsilon_{m}} \rightarrow 0$ as $m \rightarrow \infty$ and the claim is proved.

In view of (54), it follows that $v_{m} \rightarrow w$ in $L^{2}$, so that $b_{\varepsilon_{m}}\left(v_{m}, v_{m}\right)=$ $\left|v_{m}\right|_{\varepsilon_{m}} \rightarrow 1$ as $m \rightarrow \infty$. We can therefore assume that $b_{\varepsilon_{m}}\left(v_{m}, v_{m}\right) \neq 0$ for all $m \in \mathbb{N}$. Define

$$
w_{m}:=\left|v_{m}\right|_{\varepsilon_{m}}^{-1} v_{m}
$$

We have $\left|w_{m}\right|_{\varepsilon_{m}}=1$ and $b_{\varepsilon_{m}}\left(w_{m}, w_{h}^{\varepsilon_{m}}\right)=0$ for $h=1, \ldots, j-1$. Moreover,

$$
\begin{aligned}
\left\|w_{n}-w\right\|_{\varepsilon_{m}} & =\left\|\left|v_{m}\right|_{\varepsilon_{m}}^{-1} v_{m}-\left.w\right|_{\varepsilon_{m}}=\left|v_{n}\right|_{\varepsilon_{m}}^{-1}\right\| v_{m}-\left|v_{m}\right|_{\varepsilon_{m}} w \|_{\varepsilon_{m}} \\
& =\left|v_{m}\right|_{\varepsilon_{m}}^{-1}\left(\left\|v_{m}-w\right\|_{\varepsilon_{m}}+\left\|w-\left|v_{m}\right|_{\varepsilon_{m}} w\right\|_{\varepsilon_{m}}\right) \\
& =\left|v_{m}\right|_{\varepsilon_{m}}^{-1}\left(\left\|v_{m}-w\right\|_{\varepsilon_{m}}+\left(1-\left|v_{m}\right|_{\varepsilon_{m}}\right)\|w\|_{\varepsilon_{m}}\right) .
\end{aligned}
$$

Since $\left|v_{m}\right|_{\varepsilon_{m}} \rightarrow 1$ and $\|w\|_{\varepsilon_{m}} \rightarrow\|w\|_{0}$ (as $w \in H_{\mathrm{s}}^{1}$ ), it follows that

$$
\left\|w_{m}-w\right\|_{\varepsilon_{m}} \rightarrow 0 \quad \text { as } m \rightarrow \infty \text {. }
$$

Using Lemma 3.9 we obtain

$$
a_{\varepsilon_{m}}\left(w_{m}, w_{m}\right) \rightarrow a_{0}(w, w) \quad \text { as } m \rightarrow \infty .
$$

We already know that

$$
a_{\varepsilon_{m}}\left(w_{j}^{\varepsilon_{m}}, w_{j}^{\varepsilon_{m}}\right)=\lambda_{j}^{\varepsilon_{m}} \rightarrow \mu_{j}=a_{0}\left(w_{j}, w_{j}\right) \quad \text { as } m \rightarrow \infty .
$$

Moreover, for every $m$ we have

$$
a_{\varepsilon_{m}}\left(w_{j}^{\varepsilon_{m}}, w_{j}^{\varepsilon_{m}}\right) \leq a_{\varepsilon_{m}}\left(w_{m}, w_{m}\right),
$$

as $\left(w_{j}^{\varepsilon_{m}}\right)_{j \in \mathbb{N}}$ is a complete $b_{\varepsilon_{m}}$-orthonormal system of eigenvectors of the pair $\left(a_{\varepsilon_{m}}, b_{\varepsilon_{m}}\right)$. By letting $m \rightarrow \infty$, we finally obtain $a_{0}\left(w_{j}, w_{j}\right) \leq a_{0}(w, w)$. The theorem is proved.

4. Applications to reaction-diffusion equations. In this section we will apply our preceding results to reaction-diffusion equations on the squeezed domains $\Omega_{\varepsilon}=\Phi_{\varepsilon}(\Omega)$. We will, in particular, show that, as $\varepsilon \rightarrow 0^{+}$, these equations converge, in some singular sense, to a limit equation. Under some dissipativity condition we will also establish an upper semicontinuity result for the resulting family of global attractors.

Let us first recall the concept of a semiflow: 
Let $X$ be a topological space, let $D$ be an open subset of $[0, \infty[\times X$ and $\pi: D \rightarrow X$ be a continuous map. We write $x \pi t:=\pi(t, x)$ for $(t, x) \in D$. The map $\pi$ is called a local semiflow on $X$ if:

(1) For every $x \in X$ there is a number $\left.\left.\omega_{x}=\omega_{x}^{\pi} \in\right] 0, \infty\right]$ such that $(t, x) \in D$ if and only if $0 \leq t<\omega_{x}$.

(2) $x \pi 0=x$ for all $x \in X$.

(3) If $(t, x) \in D$ and $(s, x \pi t) \in D$ then $(t+s, x) \in D$ and

$$
x \pi(t+s)=(x \pi t) \pi s .
$$

If $\omega_{x}=\infty$ for every $x \in X$, then $\pi$ is called a global semiflow on $X$.

Let $J$ be an arbitrary interval in $\mathbb{R}$. A map $\sigma: J \rightarrow X$ is called a solution of $\pi$ if for all $t \in J$ and $s \in[0, \infty$ [ for which $t+s \in J$, it follows that $\sigma(t) \pi s$ is defined and $\sigma(t) \pi s=\sigma(t+s)$. If $0 \in J$ and $\sigma(0)=x$, we say that $\sigma$ is a solution through $x$. The image $\sigma(J)$ of a solution is called an orbit of $\pi$. If $J=\mathbb{R}$ then $\sigma$ is called a full solution relative to $\pi$ and its image is called a full orbit of $\pi$.

ExAmple 4.1. Let $X$ be a Banach space and $A$ be a sectorial operator in $X$ generating the family $X^{\beta}, \beta \geq 0$, of fractional power spaces. Fix an $\alpha \in\left[0,1\left[\right.\right.$ and suppose $f: X^{\alpha} \rightarrow X$ is a locally Lipschitzian map. The equation

$$
\dot{u}=-A u+f(u)
$$

defines, in the usual way, a local semiflow $\pi_{A, f}$ on $X^{\alpha}$ (see [17] or [26]). If $f$ is globally Lipschitzian on $X^{\alpha}$, then $\pi_{A, f}$ is a global semiflow.

Let $U$ be a nonempty bounded open subset of $\mathbb{R}^{l}$ with Lipschitz boundary. Let $A_{U}$ be the operator defined by the pair $(a, b)$, where $a: H^{1}(U) \times$ $H^{1}(U) \rightarrow \mathbb{R}$ is defined by

$$
a(u, v):=\int_{U} \nabla u(x) \cdot \nabla v(x) d x .
$$

Then $A_{U}$ is selfadjoint on $X=L^{2}(U)$. In particular, $A_{U}$ is sectorial on $X$, and $X^{1 / 2}=H^{1}(U)$ with equivalent norms. We interpret $A_{U}$ as the weak Laplacian on $U$ with Neumann boundary condition. More generally, consider the following reaction-diffusion equation on $U$ :

$$
\begin{array}{ll}
u_{t}=\Delta u+f(u), & t>0, x \in U, \\
\partial_{\nu} u=0, & t>0, x \in \partial U,
\end{array}
$$

where $\nu$ is the exterior normal vector field on $\partial U$ and $f: H^{1}(U) \rightarrow L^{2}(U)$ is a locally Lipschitzian map. We interpret equation (68) as being equivalent to the abstract parabolic equation

$$
\dot{u}+A_{U} u=f(u)
$$

on $H^{1}(U)$. 
In particular, using the notation of Section 3 we see that, for $\varepsilon \in] 0,1], \widetilde{A}_{\varepsilon}$ is the weak Laplacian on $\Omega_{\varepsilon}$ with Neumann boundary condition. Moreover, the operator $A_{\varepsilon}$ is sectorial on $X=L^{2}(\Omega)$ and the corresponding fractional power space $X^{\alpha}$ with $\alpha=1 / 2$ satisfies $X^{\alpha}=H^{1}(\Omega)$. If $f_{\varepsilon}: H^{1}(\Omega) \rightarrow L^{2}(\Omega)$ (resp. $\widetilde{f}_{\varepsilon}: H^{1}\left(\Omega_{\varepsilon}\right) \rightarrow L^{2}\left(\Omega_{\varepsilon}\right)$ ) is a locally Lipschitzian map we thus obtain the corresponding local semiflow $\pi_{\varepsilon, f_{\varepsilon}}:=\pi_{A_{\varepsilon}, f_{\varepsilon}}\left(\right.$ resp. $\left.\widetilde{\pi}_{\varepsilon, \tilde{f}_{\varepsilon}}:=\pi_{\widetilde{A}_{\varepsilon}, \widetilde{f}_{\varepsilon}}\right)$ on $H^{1}(\Omega)$ (resp. on $H^{1}\left(\Omega_{\varepsilon}\right)$ ). Note that, given a locally Lipschitzian map $\tilde{f}_{\varepsilon}$ : $H^{1}\left(\Omega_{\varepsilon}\right) \rightarrow L^{2}\left(\Omega_{\varepsilon}\right)$, the linear transformation $\Phi_{\varepsilon}^{*}(u):=u \circ \Phi_{\varepsilon}$ conjugates the semiflow $\widetilde{\pi}_{\varepsilon, \widetilde{f}_{\varepsilon}}$ with the semiflow $\pi_{\varepsilon, f_{\varepsilon}}$ where $f_{\varepsilon}:=\Phi_{\varepsilon}^{*} \circ \widetilde{f}_{\varepsilon} \circ\left(\Phi_{\varepsilon}^{*}\right)^{-1}$.

In particular, let $g_{\varepsilon}: \Omega_{\varepsilon} \times \mathbb{R} \rightarrow \mathbb{R}$ be a given function and $\widetilde{f}_{\varepsilon}:=\widehat{g}_{\varepsilon}$ be the Nemytskiŭ operator generated by $g_{\varepsilon}$, i.e. for $u: \Omega_{\varepsilon} \rightarrow \mathbb{R}$ set

$$
\widetilde{f}_{\varepsilon}(u)(x):=g_{\varepsilon}(x, u(x)) \quad \text { for } x \in \Omega_{\varepsilon} .
$$

Suppose $\widetilde{f}_{\varepsilon}$ restricts to a locally Lipschitzian map from $H^{1}\left(\Omega_{\varepsilon}\right)$ to $L^{2}\left(\Omega_{\varepsilon}\right)$. Then $f_{\varepsilon}:=\Phi_{\varepsilon}^{*} \circ \widetilde{f}_{\varepsilon} \circ\left(\Phi_{\varepsilon}^{*}\right)^{-1}$ is clearly given by

$$
f_{\varepsilon}(u)(x):=g_{\varepsilon}\left(\Phi_{\varepsilon}(x), u(x)\right) \quad \text { for } u \in H^{1}(\Omega) \text { and } x \in \Omega .
$$

Now note that the "limit" operator $A_{0}$ is sectorial on $X=L_{\mathrm{S}}^{2}(\Omega)$ and the corresponding fractional power space $X^{\alpha}$ with $\alpha=1 / 2$ satisfies $X^{\alpha}=$ $H_{\mathrm{s}}^{1}(\Omega)$. If $f_{0}: H_{\mathrm{s}}^{1}(\Omega) \rightarrow L_{\mathrm{s}}^{2}(\Omega)$ is a locally Lipschitzian map we thus obtain the corresponding local semiflow $\pi_{0, f_{0}}:=\pi_{A_{0}, f_{0}}$ on $H_{\mathrm{s}}^{1}(\Omega)$. Again, if $f_{0}$ is globally Lipschitzian, then $\pi_{0, f_{0}}$ is a global semiflow.

Finally, note that, for $\varepsilon \in] 0,1]$, the operator $A_{\varepsilon}$ generates a $C^{0}$-semigroup $e^{-t A_{\varepsilon}}, t \in\left[0, \infty\left[\right.\right.$, of linear operators on $L^{2}(\Omega)$, while the operator $A_{0}$ generates a $C^{0}$-semigroup $e^{-t A_{0}}, t \in\left[0, \infty\left[\right.\right.$, of linear operators on $L_{\mathrm{s}}^{2}(\Omega)$.

We can now state the following linear singular convergence result, extending [21, Theorem 4.1] to curved squeezing:

THEOREM 4.2. Let $\left(\varepsilon_{n}\right)_{n \in \mathbb{N}}$ be a sequence of positive numbers converging to zero. Let $u \in L_{\mathrm{S}}^{2}(\Omega)$ and $\left(u_{n}\right)_{n \in \mathbb{N}}$ be a sequence in $L^{2}(\Omega)$ such that $\left|u_{n}-u\right|_{L^{2}} \rightarrow 0$ as $n \rightarrow \infty$. Then for all $\left.\beta, \gamma \in\right] 0, \infty[$ with $\beta<\gamma$,

$$
\sup _{t \in[\beta, \gamma]}\left\|e^{-t A_{\varepsilon_{n}}} u_{n}-e^{-t A_{0}} u\right\|_{\varepsilon_{n}} \rightarrow 0 \quad \text { as } n \rightarrow \infty .
$$

Proof. Let $\delta>0$. Then there is an $s_{0}=s_{0}(\delta, \beta)>0$ such that $(s+1) e^{-s t}$ $<\delta$ for $s \geq s_{0}$ and $t \geq \beta$. There is a $j_{0}=j_{0}(\delta, \beta)$ such that $\lambda_{j_{0}}>s_{0}$. Thus there is an $n_{0}=n_{0}(\delta, \beta)$ such that $\lambda_{j_{0}}^{\varepsilon_{n}}>s_{0}$ for $n \geq n_{0}$. Therefore we obtain

$$
\lambda_{j}^{\varepsilon_{n}} \geq s_{0}(\delta, \beta) \quad \text { for } n \geq n_{0}(\delta, \beta) \text { and } j \geq j_{0}(\delta, \beta) .
$$

Let $P_{n}: L^{2}(\Omega) \rightarrow L^{2}(\Omega)$ be the $b_{\varepsilon_{n}}$-orthogonal projection of $L^{2}(\Omega)$ onto the span of $\left\{w_{1}^{\varepsilon_{n}}, \ldots, w_{j_{0}-1}^{\varepsilon_{n}}\right\}$. Let $P: L_{\mathrm{s}}^{2}(\Omega) \rightarrow L_{\mathrm{s}}^{2}(\Omega)$ be the $b_{0}$-orthogonal projection of $L_{\mathrm{s}}^{2}(\Omega)$ onto the span of $\left\{w_{1}, \ldots, w_{j_{0}-1}\right\}$. Let $t \in[\beta, \gamma]$. Then, for all $n \in \mathbb{N}$ and $u \in L^{2}(\Omega)$,

$$
P_{n} e^{-t A_{\varepsilon_{n}}} u=e^{-t A_{\varepsilon_{n}}} P_{n} u \in D\left(A_{\varepsilon_{n}}\right) \subset H^{1}(\Omega),
$$




$$
\left(I-P_{n}\right) e^{-t A_{\varepsilon_{n}}} u=e^{-t A_{\varepsilon_{n}}}\left(I-P_{n}\right) u \in D\left(A_{\varepsilon_{n}}\right) \subset H^{1}(\Omega) .
$$

Analogously, for all $u \in L_{\mathrm{S}}^{2}(\Omega)$,

$$
\begin{aligned}
P e^{-t A_{0}} u & =e^{-t A_{0}} P u \in D\left(A_{0}\right) \subset H_{\mathrm{s}}^{1}(\Omega), \\
(I-P) e^{-t A_{0}} u & =e^{-t A_{0}}(I-P) u \in D\left(A_{0}\right) \subset H_{\mathrm{s}}^{1}(\Omega) .
\end{aligned}
$$

It follows that

$$
\begin{aligned}
\left\|e^{-t A_{\varepsilon_{n}}} u_{n}-e^{-t A} u\right\|_{\varepsilon_{n}} & \leq\left\|P_{n} e^{-t A_{\varepsilon_{n}}} u_{n}-P e^{-t A} u\right\|_{\varepsilon_{n}} \\
& +\left\|\left(I-P_{n}\right) e^{-t A_{\varepsilon_{n}}} u_{n}\right\|_{\varepsilon_{n}}+\left\|(I-P) e^{-t A_{0}} u\right\|_{\varepsilon_{n}} .
\end{aligned}
$$

For every $\varepsilon \in[0,1]$ let $\left(\lambda_{j}^{\varepsilon}\right)_{j \in \mathbb{N}}$ be the repeated sequence of eigenvalues of $A_{\varepsilon}$ and $\left(w_{j}^{\varepsilon}\right)_{j \in \mathbb{N}}$ be a corresponding $L^{2}$-orthonormal system of eigenvectors. Write $\lambda_{j}$ and $w_{j}$ for $\lambda_{j}^{0}$ and $w_{j}^{0}$, respectively. Then

$$
\begin{aligned}
\left\|P_{n} e^{-t A_{\varepsilon_{n}}} u_{n}-P e^{-t A_{0}} u\right\|_{\varepsilon_{n}} \\
\leq \sum_{k=1}^{j_{0}-1}\left\|e^{-t \lambda_{k}^{\varepsilon_{n}}} b_{\varepsilon_{n}}\left(u_{n}, w_{k}^{\varepsilon_{n}}\right) w_{k}^{\varepsilon_{n}}-e^{-t \lambda_{k}} b_{0}\left(u, w_{k}\right) w_{k}\right\|_{\varepsilon_{n}} \\
\leq \sum_{k=1}^{j_{0}-1}\left(\left|e^{-t \lambda_{k}^{\varepsilon_{n}}} b_{\varepsilon_{n}}\left(u_{n}, w_{k}^{\varepsilon_{n}}\right)\right| \cdot\left\|w_{k}^{\varepsilon_{n}}-w_{k}\right\|_{\varepsilon_{n}}\right. \\
\left.\quad+\left|e^{-t \lambda_{k}^{\varepsilon_{n}}} b_{\varepsilon_{n}}\left(u_{n}, w_{k}^{\varepsilon_{n}}\right)-e^{-t \lambda_{k}} b_{0}\left(u, w_{k}\right)\right| \cdot\left\|w_{k}\right\|_{\varepsilon_{n}}\right) .
\end{aligned}
$$

Since $w_{k}^{\varepsilon_{n}} \rightarrow w_{k}$ and $u_{n} \rightarrow u$ in $L^{2}(\Omega)$ it follows that $b_{\varepsilon_{n}}\left(u_{n}, w_{k}^{\varepsilon_{n}}\right) \rightarrow$ $b_{0}\left(u, w_{k}\right)$ as $n \rightarrow \infty$, for $k=1, \ldots, j_{0}-1$. Moreover since $\lambda_{k}^{\varepsilon_{n}} \rightarrow \lambda_{k}$ as $n \rightarrow \infty$ we obtain

$$
\sup _{t \in[\beta, \gamma]}\left|e^{-t \lambda_{k}^{\varepsilon_{n}}}-e^{-t \lambda_{k}}\right| \rightarrow 0
$$

as $n \rightarrow \infty$, for $k=1, \ldots, j_{0}-1$. Furthermore $\left\|w_{k}^{\varepsilon_{n}}-w_{k}\right\|_{\varepsilon_{n}} \rightarrow 0$ as $n \rightarrow \infty$. Finally, $\left\|w_{k}\right\|_{\varepsilon_{n}}^{2} \leq C^{\prime}\left\|w_{k}\right\|_{0}^{2}$ for some constant $\left.C^{\prime} \in\right] 0, \infty[$ independent of $n \in \mathbb{N}$. All this, together with (77), implies that

$$
\sup _{t \in[\beta, \gamma]}\left\|P_{n} e^{-t A_{\varepsilon_{n}}} u_{n}-P e^{-t A_{0}} u\right\|_{\varepsilon_{n}} \rightarrow 0 \quad \text { as } n \rightarrow \infty .
$$

Now

$$
\begin{array}{r}
=\sum_{j=j_{0}}^{\infty}\left(\lambda_{j}^{\varepsilon_{n}}+1\right)\left|b_{\varepsilon_{n}}\left(e^{-t A_{\varepsilon_{n}}} u_{n}, w_{j}^{\varepsilon_{n}}\right)\right|^{2}=\sum_{j=j_{0}}^{\infty}\left(\lambda_{j}^{\varepsilon_{n}}+1\right)\left(e^{-t \lambda_{j}^{\varepsilon_{n}}}\right)^{2}\left|b_{\varepsilon_{n}}\left(u_{n}, w_{j}^{\varepsilon_{n}}\right)\right|^{2} \\
\leq \delta b_{\varepsilon_{n}}\left(u_{n}, u_{n}\right) \leq \delta c^{-1} C^{\prime \prime}<\infty .
\end{array}
$$


Here $C^{\prime \prime}:=\sup _{n \in \mathbb{N}}\left|u_{n}\right|_{L^{2}}^{2}<\infty$. We have used (43) and (71) above. Finally, since $(I-P) e^{-t A_{0}} u \in H_{\mathrm{S}}^{1}(\Omega)$, it follows that

$$
\begin{aligned}
& \left\|(I-P) e^{-t A_{0}} u\right\|_{\varepsilon_{n}}^{2} \leq C^{\prime}\left\|(I-P) e^{-t A_{0}} u\right\|_{0}^{2} \\
& \quad=C^{\prime} \sum_{j=1}^{\infty}\left(\lambda_{j}+1\right)\left|b_{0}\left((I-P) e^{-t A_{0}} u, w_{j}\right)\right|^{2} \leq C^{\prime} \delta|u|_{L^{2}}^{2} \leq \delta C^{\prime} C^{\prime \prime},
\end{aligned}
$$

by the same argument as in (80). Since $\delta$ is arbitrary, the conclusion of the theorem follows from (71), (79), (80) and (81).

The following concept, introduced in [6], plays a crucial role in the nonlinear singular convergence result established below:

Definition 4.3. Given $\varepsilon_{0}$ with $0<\varepsilon_{0} \leq 1$ we say that the family $\left(f_{\varepsilon}\right)_{\varepsilon \in\left[0, \varepsilon_{0}\right]}$ of maps satisfies hypothesis $(A 1)$ if:

(1) $f_{\varepsilon}: H^{1}(\Omega) \rightarrow L^{2}(\Omega)$ for every $\left.\left.\varepsilon \in\right] 0, \varepsilon_{0}\right]$, and $f_{0}: H_{\mathrm{S}}^{1}(\Omega) \rightarrow L_{\mathrm{s}}^{2}(\Omega)$.

(2) $\lim _{\varepsilon \rightarrow 0^{+}}\left|f_{\varepsilon}(u)-f_{0}(u)\right|_{L^{2}}=0$ for every $u \in H_{\mathrm{S}}^{1}(\Omega)$.

(3) For every $M \in\left[0, \infty\left[\right.\right.$ there is an $L=L_{M} \in[0, \infty[$ such that

$$
\left|f_{\varepsilon}(u)-f_{\varepsilon}(v)\right|_{L^{2}} \leq L\|u-v\|_{\varepsilon}
$$

for $\left.\varepsilon \in] 0, \varepsilon_{0}\right]$ and $u, v \in H^{1}(\Omega)$ satisfying $\|u\|_{\varepsilon},\|v\|_{\varepsilon} \leq M$. Moreover,

$$
\left|f_{0}(u)-f_{0}(v)\right|_{L^{2}} \leq L\|u-v\|_{0}
$$

for $u, v \in H_{\mathrm{S}}^{1}(\Omega)$ satisfying $\|u\|_{0},\|v\|_{0} \leq M$.

The following simple extension of [6, Proposition 2.6] shows how we can obtain, in applications, families of maps satisfying hypothesis $(A 1)$ :

Proposition 4.4. Let $G: \mathbb{R} \times \mathbb{R}^{l} \times \mathbb{R} \rightarrow \mathbb{R},(\varepsilon, x, \xi) \mapsto G(\varepsilon, x, \xi)$, be a $C^{1}$-function for which there are constants $\beta, \delta, C \in[0, \infty[$ such that for all $(\varepsilon, x, \xi) \in \mathbb{R} \times \mathbb{R}^{l} \times \mathbb{R}$,

(1) $\left|\partial_{\varepsilon} G(\varepsilon, x, \xi)\right| \leq C\left(1+|\xi|^{\beta}\right)$;

(2) $\left|\nabla_{x} G(\varepsilon, x, \xi)\right| \leq C\left(1+|\xi|^{\beta}\right)$;

(3) $\left|\partial_{\xi} G(\varepsilon, x, \xi)\right| \leq C\left(1+|\xi|^{\delta}\right)$.

If $l>2$ then assume also that $\beta \leq 2^{*} / 2$ and $\delta \leq 2^{*} / 2-1$, where $2^{*}:=$ $2 l /(l-2)$. For $\varepsilon>0$ and $u \in H^{1}(\Omega)$ define $f_{\varepsilon}(u): \Omega \rightarrow \mathbb{R}$ by

$$
f_{\varepsilon}(u)(x):=G\left(\varepsilon, \Phi_{\varepsilon}(x), u(x)\right) \quad \text { for } x \in \Omega .
$$

Furthermore, for $u \in H_{\mathrm{s}}^{1}(\Omega)$ define $f_{0}(u): \Omega \rightarrow \mathbb{R}$ by

$$
f_{0}(u)(x):=G(0, \phi(x), u(x)) \quad \text { for } x \in \Omega \text {. }
$$

Then the family $\left(f_{\varepsilon}\right)_{\varepsilon \in[0,1]}$ satisfies hypothesis $(A 1)$.

Proof. That $f_{0}$ maps $H_{\mathrm{s}}^{1}(\Omega)$ into $L_{\mathrm{s}}^{2}(\Omega)$ follows by a modification of the proof of [21, Theorem 5.3]. (Cf. also the proof of Proposition 3.5.) All the other assertions follow by an application of the Sobolev imbedding theorems, 
the Hölder inequality and the mean value theorem. The details are left to the reader.

REMARK 4.5. Proposition 4.4 can be generalized to functions $G$ taking values in $\mathbb{R}^{p}$ and having the argument $\xi$ lying in $\mathbb{R}^{p}$. This allows applications to systems of reaction-diffusion equations.

We can also state the following nonlinear singular convergence theorem, generalizing [21, Theorem 5.1] and [6, Theorem 2.13]:

THEOREM 4.6. Let $\left(f_{\varepsilon}\right)_{\varepsilon \in[0,1]}$ satisfy hypothesis $(A 1)$ and $\left(\varepsilon_{n}\right)_{n \in \mathbb{N}}$ be a sequence of positive numbers converging to zero. Write $\pi_{n}:=\pi_{A_{\varepsilon_{n}}, f_{\varepsilon_{n}}}$ and $\pi:=\pi_{A_{0}, f_{0}}$. Let $u \in H_{\mathrm{s}}^{1}(\Omega)$ and let $\left(u_{n}\right)_{n \in \mathbb{N}}$ be a sequence in $H^{1}(\Omega)$ such that $\left|u_{n}-u\right|_{L^{2}} \rightarrow 0$ as $n \rightarrow \infty$. Let $\left.b \in\right] 0, \infty\left[\right.$ and suppose that $u_{n} \pi_{n}$ s and $u \pi s$ are defined for all $s \in[0, b]$ and $n \in \mathbb{N}$, and

$$
\sup _{n \in \mathbb{N}} \sup _{s \in[0, b]}\left\|u_{n} \pi_{n} s\right\|_{\varepsilon_{n}} \leq M \quad \text { and } \quad \sup _{n \in \mathbb{N}} \sup _{s \in[0, b]}\|u \pi s\|_{\varepsilon_{n}} \leq M
$$

for some constant $M \in\left[0, \infty\left[\right.\right.$. Finally, let $\left.\left.t_{0} \in\right] 0, b\right]$ and $\left(t_{n}\right)_{n \in \mathbb{N}}$ be a sequence in $] 0, b]$ converging to $t_{0}$. Then

$$
\left\|u_{n} \pi_{n} t_{n}-u \pi t_{0}\right\|_{\varepsilon_{n}} \rightarrow 0 \quad \text { as } n \rightarrow \infty .
$$

Proof. First note that there is a constant $C_{1}>0$ such that for all $\varepsilon \in$ ]0,1], $r \in] 0, \infty\left[\right.$ and $v \in L^{2}(\Omega)$,

$$
\left\|e^{-A_{\varepsilon} r} v\right\|_{\varepsilon} \leq C_{1}\left(r^{-1 / 2}+1\right)|v|_{L^{2}}
$$

and, for all $v \in L_{\mathrm{s}}^{2}(\Omega)$,

$$
\left\|e^{-A_{0} r} v\right\|_{\varepsilon} \leq C_{1}\left(r^{-1 / 2}+1\right)|v|_{L^{2}} .
$$

Let $L=L_{M}$ be as in hypothesis $(A 1)$. For every $t \in[0, b]$ we have, by the variation-of-constants formula,

$$
\begin{aligned}
u_{n} \pi_{n} t-u \pi t= & e^{-A_{\varepsilon_{n}} t} u_{n}-e^{-A_{0} t} u+\int_{0}^{t} e^{-A_{\varepsilon_{n}}(t-s)}\left(f_{\varepsilon_{n}}\left(u_{n} \pi_{n} s\right)-f_{\varepsilon_{n}}(u \pi s)\right) d s \\
& +\int_{0}^{t}\left(e^{-A_{\varepsilon_{n}}(t-s)} f_{\varepsilon_{n}}(u \pi s)-e^{-A_{0}(t-s)} f_{0}(u \pi s)\right) d s .
\end{aligned}
$$

Define $g_{n}:[0, b] \times[0, b] \rightarrow \mathbb{R}$ by

$$
g_{n}(t, s)= \begin{cases}\left\|e^{-A_{\varepsilon_{n}}(t-s)} f_{\varepsilon_{n}}(u \pi s)-e^{-A_{0}(t-s)} f_{0}(u \pi s)\right\|_{\varepsilon_{n}} & \text { if } 0<s<t, \\ 0 & \text { otherwise }\end{cases}
$$

The function $g_{n}$ restricted to the set of $(s, t)$ with $0<s<t$ is continuous by $(A 1)$ and Theorem 4.2. Thus $g_{n}$ is measurable on $[0, b] \times[0, b]$. By Fubini's theorem the function

$$
c_{n}(t):=\int_{0}^{b} g_{n}(t, s) d s=\int_{0}^{t} g_{n}(t, s) d s
$$


is a.e. defined and measurable on $[0, b]$. Set

$$
a_{n}(t):= \begin{cases}\left\|e^{-A_{\varepsilon_{n}} t} u_{n}-e^{-A_{0} t} u\right\|_{\varepsilon_{n}}+c_{n}(t) & \text { for } t \in] 0, b], \\ 0 & \text { for } t=0 .\end{cases}
$$

It follows that $a_{n}$ is measurable on $[0, b]$. Using (82) and (83) we obtain

$$
\left|g_{n}(t, s)\right| \leq 2 C_{2} C_{1}\left((t-s)^{-1 / 2}+1\right) \quad \text { whenever } 0<s<t \leq b
$$

where

$$
C_{2}:=\max \left\{\sup _{s \in[0, b]} \sup _{n \in \mathbb{N}}\left|f_{\varepsilon_{n}}(u \pi s)\right|_{L^{2}}, \sup _{s \in[0, b]}\left|f_{0}(u \pi s)\right|_{L^{2}}\right\} .
$$

Let $L=L_{M}$ be as in $(A 1)$. Note that, by $(A 1)$,

$$
\begin{aligned}
\left|f_{\varepsilon_{n}}(u \pi s)\right|_{L^{2}} & \leq\left|f_{\varepsilon_{n}}(u \pi s)-f_{\varepsilon_{n}}(u)\right|_{L^{2}}+\left|f_{\varepsilon_{n}}(u)\right|_{L^{2}} \\
& \leq L|u \pi s-u|_{\varepsilon_{n}}+\left|f_{\varepsilon_{n}}(u)\right|_{L^{2}} \leq M^{\prime}
\end{aligned}
$$

for some constant $M^{\prime}<\infty$, independent of $n \in \mathbb{N}$ and $s \in[0, b]$. Similarly, we may assume that

$$
\left|f_{0}(u \pi s)\right|_{L^{2}} \leq M^{\prime}, \quad s \in[0, b] .
$$

This shows that $C_{2}<\infty$.

If $0<s<t_{0}$ then for some $n_{0}$ and some $\beta>0, t_{n}-s>\beta$ for $n \geq n_{0}$. By Theorem 4.2, $g_{n}\left(t_{n}, s\right) \rightarrow 0$. If $0<t<s$ then for some $n_{0}, t_{n}<s$ and so $g_{n}\left(t_{n}, s\right)=0$ for all $n \geq n_{0}$. Again $g_{n}\left(t_{n}, s\right) \rightarrow 0$. It follows from the dominated convergence theorem that $c_{n}\left(t_{n}\right) \rightarrow 0$. Thus, again using Theorem 4.2 we obtain $a_{n}\left(t_{n}\right) \rightarrow 0$. In particular,

$$
\left.\left.a_{n}(t) \rightarrow 0 \quad \text { for all } t \in\right] 0, b\right] .
$$

Furthermore, the definition of $a_{n}$ clearly implies that

$$
\left.\left.a_{n}(t) \leq C_{3}\left(t^{-1 / 2}+1\right) \quad \text { for } t \in\right] 0, b\right] .
$$

An application of Henry's inequality ([17, Lemma 7.1.1]) implies that

$$
\left.\left.\left\|u_{n} \pi_{n} t-u \pi t\right\|_{\varepsilon_{n}} \leq a_{n}(t)+\int_{0}^{t} \varrho(t-s) a_{n}(s) d s \quad \text { for } t \in\right] 0, b\right],
$$

where

$$
\varrho(x):=\sum_{n=1}^{\infty} \frac{(L \Gamma(\beta))^{n}}{\Gamma(n \beta)} x^{n \beta-1}
$$

with $\beta:=1 / 2$. The function $\varrho:] 0, \infty[\rightarrow] 0, \infty[$ is well defined and continuous on $] 0, \infty[$ and it satisfies the estimate

$$
\left.\left.\varrho(x) \leq C_{4} x^{-1 / 2}+C_{4} \quad \text { for } x \in\right] 0, b\right] .
$$

Fix a $\delta_{0}$ with $0<\delta_{0}<t$ and let $\delta>0$ with $2 \delta<\delta_{0}$. There is an $n_{0}=n_{0}(\delta)$ such that $\left|t_{n}-t\right|<\delta$ for $n \geq n_{0}$. Therefore for all such $n$ and all $s \in[0, t-2 \delta]$ 
it follows that $t_{n}-s>\delta$ so $\varrho\left(t_{n}-s\right) \leq C_{4} \delta^{-1 / 2}+C_{4}$. Thus

$$
\left.\left.\varrho\left(t_{n}-s\right) a_{n}(s) \leq C_{5}\left(s^{-1 / 2}+1\right) \quad \text { for } s \in\right] 0, t-2 \delta\right] .
$$

Therefore (84) and the dominated convergence theorem show that

$$
\int_{0}^{t-2 \delta} \varrho\left(t_{n}-s\right) a_{n}(s) d s \rightarrow 0 .
$$

On the other hand, for $s \in\left[t-2 \delta, t_{n}\right]$ we have $s \geq t-\delta_{0}>0$ so $a_{n}(s) \leq C_{6}$. Therefore

$$
\int_{t-2 \delta}^{t_{n}} \varrho\left(t_{n}-s\right) a_{n}(s) d s \leq C_{7}\left(\delta^{1 / 2}+\delta\right) .
$$

Since $\delta<\delta_{0}$ is arbitrary, it follows that

$$
\int_{0}^{t_{n}} \varrho\left(t_{n}-s\right) a_{n}(s) d s \rightarrow 0 .
$$

Consequently,

$$
\left\|u_{n} \pi_{n} t_{n}-u \pi t_{n}\right\|_{\varepsilon_{n}} \rightarrow 0
$$

To conclude the proof, note that, for some constant $\left.C^{\prime} \in\right] 0, \infty[$ independent of $n \in \mathbb{N}$,

$$
\left\|u \pi t_{n}-u \pi t_{0}\right\|_{\varepsilon_{n}} \leq C^{\prime}\left\|u \pi t_{n}-u \pi t_{0}\right\|_{0} \rightarrow 0
$$

COROLlaRY 4.7. Let $\left(f_{\varepsilon}\right)_{\varepsilon \in[0,1]},\left(\varepsilon_{n}\right)_{n \in \mathbb{N}}, \pi_{n}:=\pi_{A_{\varepsilon_{n}}, f_{\varepsilon_{n}}}$ and $\pi:=\pi_{A_{0}, f_{0}}$ be as in Theorem 4.6. Let $C \in\left[0, \infty\left[\right.\right.$ and for every $n \in \mathbb{N}$ let $\sigma_{n}: \mathbb{R} \rightarrow$ $H^{1}(\Omega)$ be a solution of $\pi_{n}$ such that

$$
\sup _{t \in \mathbb{R}}\left\|\sigma_{n}(t)\right\|_{\varepsilon_{n}} \leq C .
$$

Assume also that $\pi$ is a global semiflow. Then there is a subsequence of $\left(\sigma_{n}\right)_{n}$, still denoted by $\left(\sigma_{n}\right)_{n}$, and a solution $\sigma: \mathbb{R} \rightarrow H_{\mathrm{s}}^{1}$ of $\pi$ such that

$$
\left\|\sigma_{n}(t)-\sigma(t)\right\|_{\varepsilon} \rightarrow 0 \quad \text { for every } t \in \mathbb{R} .
$$

Proof. Let $\left(u_{n}\right)_{n}$ be a sequence in $H^{1}(\Omega)$ such that

$$
\sup _{n \in \mathbb{N}}\left\|u_{n}\right\|_{\varepsilon_{n}} \leq C .
$$

It has a subsequence, again denoted by $\left(u_{n}\right)_{n}$, such that $\left(u_{n}\right)_{n}$ converges weakly in $H^{1}(\Omega)$ and strongly in $L^{2}(\Omega)$ to some $u \in H^{1}(\Omega)$. In view of (85) we have $P(\cdot) \nabla u_{n} \rightarrow 0$ in $L^{2}(\Omega)$, which easily implies that $P(\cdot) \nabla u=0$, i.e. $u \in H_{\mathrm{s}}^{1}(\Omega)$. Applying this to the sequences $\left(\sigma_{n}(-k)\right)_{n}$ for every $k \in \mathbb{N}_{0}$ and using Cantor's diagonal procedure we easily obtain the existence of a subsequence of $\left(\sigma_{n}\right)_{n}$, still denoted by $\left(\sigma_{n}\right)_{n}$, and a sequence $v(-k) \in H_{\mathrm{s}}^{1}(\Omega)$, $k \in \mathbb{N}_{0}$, such that for every $k \in \mathbb{N}_{0}$ the subsequence $\left(\sigma_{n}(-k)\right)$ converges weakly in $H^{1}(\Omega)$ and strongly in $L^{2}(\Omega)$ to $v(-k)$. It follows that

$$
\tau_{k}(t):=v(-k) \pi(t+k)
$$


is well defined for all $t \in\left[-k, \infty\left[\right.\right.$. For every $k \in \mathbb{N}_{0}$ and every $\left.t \in\right]-k, \infty[$,

$$
\sigma_{n}(t)=\sigma_{n}(-k) \pi_{n}(t+k)
$$

so by Theorem 4.6,

$$
\left\|\sigma_{n}(t)-\tau_{k}(t)\right\|_{\varepsilon_{n}} \rightarrow 0 .
$$

In particular, if $l, k \in \mathbb{N}_{0}$ with $l>k$, this implies that $\tau_{l}(t)=\tau_{k}(t)$ for $t \in\left[-k, \infty\left[\right.\right.$. It follows that there exists a unique function $\sigma: \mathbb{R} \rightarrow H_{\mathrm{s}}^{1}(\Omega)$ such that $\sigma(t)=\tau_{k}(t)$ for $t \in[-k, \infty[$. Consequently, $\sigma$ is the required solution of $\pi$.

REMARK 4.8. Note that Theorem 4.6 and its corollary remain valid, with obvious changes and the same proof, if the operators $A_{\varepsilon}, \varepsilon \in[0,1]$, are replaced by "decoupled" systems of operators $B_{\varepsilon}:\left(D\left(A_{\varepsilon}\right)\right)^{p} \rightarrow\left(L^{2}(\Omega)\right)^{p}, \varepsilon \in$ ] $0,1]$, and $B_{0}:\left(D\left(A_{0}\right)\right)^{p} \rightarrow\left(L_{\mathrm{s}}^{2}(\Omega)\right)^{p}$, with $B_{\varepsilon}\left(u_{1}, \ldots, u_{p}\right):=\left(d_{1} A_{\varepsilon}, \ldots, d_{p} A_{\varepsilon}\right)$, where $\left(d_{1}, \ldots, d_{p}\right)$ is a fixed vector in $\mathbb{R}^{p}$ with positive components. Thus Theorem 4.6 and its corollary are applicable to systems of reaction-diffusion equations on squeezed domains. Cf. also Remark 4.5.

We will now show that, under the usual dissipativeness assumption, each semiflow $\pi_{\varepsilon}, \varepsilon \in[0,1]$, has a global attractor $\mathcal{A}_{\varepsilon}$ and that this family of attractors is upper semicontinuous at $\varepsilon=0$. This generalizes, to curved squeezing, the corresponding results from [21]. We assume that the reader is familiar with some basic theory of attractors for evolution equations, as expounded in the monographs [12], [19] or [9].

TheOREM 4.9. Assume that $G: \mathbb{R} \rightarrow \mathbb{R}$ is a $C^{1}$-function such that

$$
\left|G^{\prime}(s)\right| \leq C\left(|s|^{\beta}+1\right) \quad \text { for } s \in \mathbb{R}
$$

where $C, \beta \in[0, \infty[$ are constants. If $l>2$ then assume in addition that $\beta \leq 2^{*} / 2-1$, where $2^{*}=2 l /(l-2)>2$. Moreover, suppose that $G$ is dissipative in the sense that

$$
\limsup _{|s| \rightarrow \infty} G(s) / s \leq-\zeta \quad \text { for some } \zeta>0 .
$$

For $\varepsilon \in] 0,1]$ define $f_{\varepsilon}: H^{1}(\Omega) \rightarrow L^{2}(\Omega)$ by $f_{\varepsilon}(u)=G \circ u$. Furthermore, define $f_{0}: H_{\mathrm{s}}^{1}(\Omega) \rightarrow L_{\mathrm{s}}^{2}(\Omega)$ by $f_{0}(u)=G \circ u$. For $\varepsilon \in[0,1]$ let $\pi_{\varepsilon}:=\pi_{A_{\varepsilon}, f_{\varepsilon}}$, and let $\mathcal{A}_{\varepsilon}$ be the union of all full bounded orbits of $\pi_{\varepsilon}$. Then, for all $\varepsilon \in[0,1]$, $\pi_{\varepsilon}$ is a global semiflow and the set $\mathcal{A}_{\varepsilon}$ is nonempty, compact, connected in $H^{1}(\Omega)$. Furthermore, $\mathcal{A}_{\varepsilon}$ attracts every set $B$ which is bounded in $H^{1}(\Omega)$ for $\varepsilon \in] 0,1]$ and in $H_{\mathrm{s}}^{1}(\Omega)$ for $\varepsilon=0$. In other words, for every such $B$,

$$
\lim _{t \rightarrow \infty} \sup _{u \in B} \inf _{v \in \mathcal{A}_{\varepsilon}}\left\|u \pi_{\varepsilon} t-v\right\|_{\varepsilon}=0 .
$$

The family $\left(\mathcal{A}_{\varepsilon}\right)_{\varepsilon \in\left[0, \varepsilon_{0}\right]}$ is upper semicontinuous at $\varepsilon=0$ with respect to the family $\left(\|\cdot\|_{\varepsilon}\right)$ of norms, i.e.

$$
\lim _{\varepsilon \rightarrow 0^{+}} \sup _{u \in \mathcal{A}_{\varepsilon}} \inf _{v \in \mathcal{A}_{0}}\|u-v\|_{\varepsilon}=0
$$


Proof. Proposition 4.4 implies that the maps $f_{\varepsilon}$ and the local semiflows $\pi_{\varepsilon}, \varepsilon \in[0,1]$, are well defined. All the other assertions follow exactly like those of [21, Theorems 5.8 and 5.10]. In particular, the upper semicontinuity of the attractor family follows by an application of Corollary 4.7. We omit the easy details.

\section{References}

[1] R. Abraham, J. E. Marsden and T. Ratiu, Manifolds, Tensor Analysis, and Applications, Springer, New York, 1988.

[2] J. Arrieta, Rates of eigenvalues on a dumbbell domain. Simple eigenvalue case, Trans. Amer. Math. Soc. 347 (1995), 3503-3531.

[3] -, Neumann eigenvalue problems on exterior perturbation of the domain, J. Differential Equations 118 (1995), 54-103.

[4] -, Spectral behavior and upper semicontinuity of attractors, International Conference on Differential Equations, Vol. 1, 2 (Berlin, 1999), World Sci., River Edge, NJ, 2000, 615-621.

[5] M. Berger and B. Gostiaux, Differential Geometry: Manifolds, Curves and Surfaces, Springer, New York, 1988.

[6] M. C. Carbinatto and K. P. Rybakowski, Conley index continuation and thin domain problems, Topol. Methods Nonlinear Anal. 16 (2000), 201-251.

[7] -, 一, On convergence, admissibility and attractors for damped wave equations on squeezed domains, Proc. Roy. Soc. Edinburgh Sect. A, to appear.

[8] - - - On a general Conley index continuation principle for singular perturbation problems, Ergodic Theory Dynam. Systems, to appear.

[9] J. W. Cholewa and T. Dłotko, Global Attractors in Abstract Parabolic Problems, Cambridge Univ. Press, Cambridge, 2000.

[10] P. G. Ciarlet, Mathematical Elasticity, Volume II: Theory of Plates, Elsevier, Amsterdam, 1997.

[11] I. S. Ciuperca, Reaction-diffusion equations on thin domains with varying order of thinness, J. Differential Equations 126 (1996), 244-291.

[12] J. Hale, Asymptotic Behavior of Dissipative Systems, Math. Surveys Monogr. 25, Amer. Math. Soc., Providence, 1988.

[13] J. Hale and G. Raugel, Reaction-diffusion equations on thin domains, J. Math. Pures Appl. (9) 71 (1992), 33-95.

[14] - - - A damped hyperbolic equation on thin domains, Trans. Amer. Math. Soc. 329 (1992), 185-219.

[15] - - - Upper semicontinuity of the attractor for a singularly perturbed hyperbolic equation, J. Differential Equations 73 (1988), 197-214.

[16] J. Hale and J. Vegas, A nonlinear parabolic equation with varying domain, Arch. Rational Mech. Anal. 86 (1984), 99-123.

[17] D. Henry, Geometric Theory of Semilinear Parabolic Equations, Springer, Berlin, 1981.

[18] S. Jimbo and Y. Morita, Remarks on the behavior of certain eigenvalues on a singularly perturbed domain with several thin channels, Comm. Partial Differential Equations 17 (1992), 523-552.

[19] O. Ladyzhenskaya, Attractors for Semigroups and Evolution Equations, Cambridge Univ. Press, Cambridge, 1991. 
[20] S. M. Oliva, Reaction-diffusion equations on domains with thin channels, J. Differential Equations 123 (1995), 437-479.

[21] M. Prizzi and K. P. Rybakowski, The effect of domain squeezing upon the dynamics of reaction-diffusion equations, ibid. 173 (2001), 271-320.

[22] - - - Some recent results on thin domain problems, Topol. Methods Nonlinear Anal. 14 (1999), 239-255.

[23] —, - Inertial manifolds on squeezed domains, J. Dynamics Differential Equations, to appear.

[24] —, - Inertial manifolds on high-dimensional thin domains, submitted.

[25] G. Raugel, Dynamics of partial differential equations on thin domains, in: R. Johnson (ed.), Dynamical Systems (Montecatini Terme, 1994), Lecture Notes in Math. 1609, Springer, Berlin, 1995, 208-315.

[26] K. P. Rybakowski, The Homotopy Index and Partial Differential Equations, Springer, Berlin, 1987.

Dipartimento di Scienze Matematiche

DISCAFF

Università degli Studi di Trieste

Via Valerio, 12/b

34100 Trieste, Italy

E-mail: prizzi@mathsun1.univ.trieste.it

Viale Ferrucci, 33

28100 Novara, Italy

Fachbereich Mathematik

Universität Rostock

Universitätsplatz 1

18055 Rostock, Germany

E-mail: krzysztof.rybakowski@mathematik.uni-rostock.de 